$\begin{array}{ll}\text { Research Square } & \begin{array}{l}\text { Preprints are preliminary reports that have not undergone peer review. } \\ \text { They should not be considered conclusive, used to inform clinical practice, } \\ \text { or referenced by the media as validated information. }\end{array}\end{array}$

\title{
Vermicompost Assisted Phytoremediation of Heavy Metal Contaminated Soil in Madaka District, Nigeria Using Melissa officinalis L (Lemon balm) and Sida acuta (Stubborn weed)
}

sesan abiodun aransiola ( $\sim$ blessedabiodun@gmail.com )

Federal University of Technology Minna - Bosso Campus

Udeme Joshua Joshia ljah

Federal University of Technology Minna

Olabisi Peter Abioye

Federal University of Technology Minna

Jeremiah David Bala

Federal University of Technology Minna - Bosso Campus

Research Article

Keywords: Microbial, Phytoremediation, Vermicompost, Soil, Heavy metal, Contamination, Manure

Posted Date: March 5th, 2021

DOI: https://doi.org/10.21203/rs.3.rs-276415/v1

License: (a) (1) This work is licensed under a Creative Commons Attribution 4.0 International License. Read Full License 


\section{Abstract}

This research was designed to clean-up through an environmentally friendly techniques, a polluted environment of Madaka District of Shikira. Physicochemical properties of the soil were done using standard methods. Chicken dropping vermicompost (CDV) and goat manure vermicompost (GMV) were produced by standard method to assist the phytoremediation process with plant growth promoting bacteria (PGPB). Canonical discriminant functions of the heavy metals were done. For the first location (AK) the plant parts of $M$. officinalis $\mathrm{L}$ mopped up heavy metals, the concentration of $\mathrm{Cd}$, As, $\mathrm{Pb}$ in plant parts varied from 0.007 to $0.33 \mathrm{mg} / \mathrm{kg}$, As from 0.09 to $4.39 \mathrm{mg} / \mathrm{kg}$ and $\mathrm{Pb}$ from 0.07 to $10.35 \mathrm{mg} / \mathrm{kg}$ respectively while the concentration of heavy metals in S. acuta parts had Cd, As, Pb varied from 0.002 to $0.43 \mathrm{mg} / \mathrm{kg}$, As from 0.27 to $3.79 \mathrm{mg} / \mathrm{kg}$ and Pb from 1.68 to $10.7 \mathrm{mg} / \mathrm{kg}$ respectively. The second location (AM) also had the two plants mopping up heavy metals at different concentrations. the concentration of $\mathrm{Cd}, \mathrm{As}$, $\mathrm{Pb}$ in $M$. officinalis $\mathrm{L}$ parts varied from 0.03 to $0.41 \mathrm{mg} / \mathrm{kg}$, As from 0.65 to $4.65 \mathrm{mg} / \mathrm{kg}$ and $\mathrm{Pb}$ from 1.93 to $11.49 \mathrm{mg} / \mathrm{kg}$ respectively while the concentration of heavy metals in $S$. acuta parts had Cd, As, $\mathrm{Pb}$ varied from 0.06 to $0.66 \mathrm{mg} / \mathrm{kg}$, As from 0.68 to $4.64 \mathrm{mg} / \mathrm{kg}$ and $\mathrm{Pb}$ from 1.53 to $11.53 \mathrm{mg} / \mathrm{kg}$ respectively. Melissa offinalis $\mathrm{L}$ and Sida acuta were found most suitable for phytoextraction of sites contaminated with $\mathrm{Cd}$, As and $\mathrm{Pb}$ because both of them have their bioconcentration factor (BCF), translocation factor (TF) and biological accumulation coefficient (BAC) to be $₫ 1$ while both were also scored as phytostabilizer because they have BCF $₫ 1$ and TF $₫ 1$. Soil structural changes pre and post remediation were determined through x-ray fluorescence (XRF spectroscopy) and scanning electron microscopy (SEM) analyses.

\section{Introduction}

Currently, pollution is one of the most important problems around the world where several world inhabitants suffer health problems related to mining, atmospheric pollutant and industry (Martinez et al. 2004). Mining amongst human activities has been recognized to cause major negative impact on the wholistic quality of the environment (Donkor et al. 2005; Acosta et al. 2011). Human anthropogenic activities for example mining causes the destruction of natural ecosystems by altering soil, vegetations soil microorganisms. The unwanted release of environmental pollutants predisposed by mining activities had reached an alarming proportion that deserves attention (Abiya et al. 2019; Ullah et al. 2015) The presence of these heavy metals in soil and water bodies is known to significantly deteriorate the quality of such soil and waters (Amarlal et al. 2006; Aransiola, et al. 2021). Zamfara State lead poisoning is one of the major heavy metal incidences in the Nigerian records that claimed the lives of over 500 children within seven months. Between January and July, 2010 , illegal miners from seven villages of Bukkuyum and Gummi Local Governments in Zamfara State brought rocks containing gold ore into the villages from smallscale mining operations; however, the villagers did not know that the ore also contained extremely high levels of lead. The ore was crushed inside village compounds, spreading lead dust throughout the community. These led to the death of many villagers, mainly children (Galadima and Garba 2011). Heavy metals are of concern due to their capacity to induce harmful and toxic effects on plants and animals. Some metals are known to have importance in growth at minute quantities (Cu, Fe, Mn, Zn, and Mo), but surplus levels negatively affect growth and development (Alirzayeva et al. 2017; Ali et al. 2021) They are one of the most persistent pollutants in soil and water (Aransiola et al. 2021).

Vermicomposting is a green technique that produces vermicompost from different types of organic wastes using specific earthworm species. It helps farmers to reduce their use of chemical fertilizers and the overall production costs. Vermicompost is considered an alternative to chemical additives in agricultural crop production that reduces economic costs, while producing healthier organic products for consumers and enriching the environment (Kaplan 2016). Some communities in Niger State such as Shikira community in Rafi Local Government Area (RLGA), where there is large gold deposit and through a series of mining activities have their environment heavily polluted with lead $(\mathrm{Pb})$ and other metals (Ikhumetse et al. 2019). This lead poison led to the death of 28 children in the year 2015 as reported by the Federal Ministry of Health FMH, Nigeria (FMH 2015). Excessive metal concentration in soils pose significant hazard to human in particular, and to the environment in general. Contamination of soils with toxic metals has often resulted from uncontrolled human activities especially those related to mining as practised in Madaka District, RLGA, Niger State, Nigeria (FMH 2015) especially those related to mining. There is an urgent need to develop an eco-friendly remediation technology to effectively remove the contaminating metals from the polluted soil of these communities. Therefore, Vermicomposts were employed in this study in an assisted capacity to restore the polluted soil using S. acuta and Melissa officinalis L

\section{Materials And Methods}

\section{Study area}

The study site was Madaka District, Shikira community comprising two settlements - Angwan Magiro (AM) and Angwan Kawo (AK). Shikira is located by the Eastern part of Kagara town, the headquarters of Rafi Local Government Area of Niger State, Nigeria (Figure 1). Niger State is located between longitude $3^{\circ} 30^{\prime}$ $\mathrm{E}$ and $7^{\circ} 30^{\prime} \mathrm{E}$ and latitude $8^{\circ} 10^{\prime} \mathrm{N}$ and $10^{\circ} 30^{\prime} \mathrm{N}$ (Figure 1). The site was selected based on the report of the lead poisoning occurrence in May, $2015 \mathrm{due}$ to artisanal mining activities (FMH, 2015). Madaka people are predominantly farmers while few are nomads. Mining activities have been going on in Madaka district for years and this involves both the indigenes and foreigners (Ikhumetse et al., 2019)

\section{Collection and Processes of Samples}

Plants and Heavy metal polluted soil for this experiment were collected from the mining sites of Shikira community comprising Angwan Magiro and Angwan Kawo, Rafi Local Government Area, Niger State, Nigeria, from a depth of 0-15 cm with clean stainless-steel shovel and transported in polythene bags to Federal University of Technology, Minna, for further analysis. These two major sites where pollution of heavy metals and extensive mining activities have taken place were selected for this study. In each of the sites, a $100 \mathrm{~m}$ by $100 \mathrm{~m}$ plot were demarcated and the two repeated herbaceous plant species (Sida acuta and Melissa officinalis $\mathrm{L}$ ) contained in the plots were selected for this study.

\section{Physicochemical Properties}


The physicochemical properties of the soil were carried following using standard methods as described by different authors. Soil pH was determined using the method of Eckerts and Sims (1995), organic carbon and the exchangeable cations were determined by the method of Walkley and Black (1934) and Agbenin

(1995). The method of Black (1965) and Agbenin (1995) were used to determined the total nitrogen while the soil particle size was done using the methods of Bouyoucos (1962) and USEPA (1996), available phosphorus was determined by the method of Bray and Kurtz (1945) and Nordberg et al. (2007)

\section{Collection of Vermicompost Materials and Preparation of Chicken Dropping (CDV) and Goat Manure Vermicompost (GMV)}

The wastes as the raw material for the vermicompost (a) Dried neem leaves that was used for the bottom layer of the vermicompost were collected within the environment of the Federal University of Technology, Minna, Niger State, Nigeria. These were collected in a clean plastic container for the set-up. (b) Rice straw, this was collected at mini rice mill factory located in Sauka Kahuta area, Minna, Niger State, Nigeria. The collection of this was taken into a clean sack, stored and ready for the set-up. (c) Vegetable wastes, these wastes were collected at vegetable market, Kasangwari, market, Mobil, Minna, Niger State, Nigeria. (d) Goat dung was collected at the goat market located at Kasangwari market, Minna, Niger State, Nigeria. (e) Chicken dropping was collected from Royal Splendour Integrated School farm, Mandela area, Minna, Niger State, Nigeria. (f) Exotic varieties of earthworm (Eisenia foetida) were purchased from fishermen at riverine village of Taji, Lokoja Local Government Area, Kogi State, Nigeria. About $2 \mathrm{~kg}$ were used in vermicomposting.

The vermin-compost was produced from chicken dropping and vegetable waste. In this process, dried neem leaves ( $1 \mathrm{~kg})$, chopped rice straw (2 kg) was added with the vegetable wastes (half a $500 \mathrm{~g}$ ) and chicken dropping $(4 \mathrm{~kg})$. Water $(2 \mathrm{~L})$ was added and one kilogramme (1 kg) of exotic varieties of earthworms (Eisenia foetida) (Figure 2a) was spread on bedding materials in the plastic worm composter. This set-up was monitored for 90 days and water (2 $L$ at 2 days interval) was introduced to curb dryness of the bed. The physicochemical parameters were measured during vermin-composting as described in soil analysis and these include; $\mathrm{pH}$, organic matter, total nitrogen, available nitrogen, total phosphorus, sodium, magnesium, iron, zinc, manganese and copper (Figures 2a \& b). After 90 days, chicken vermicompost was collected, air dried, and a portion of it was taken for nutrient analysis (Jadia and Fulekar 2008). The nutrients in dried sample of vermin-compost was digested with concentrated nitric acid and $30 \%$ hydrogen peroxide and then determined by atomic absorption spectrophotometry (AAS). This set-up was replicated in another vermin composter where goat dung replaced the chicken droppings (Figures $2 \mathrm{~d} \& \mathrm{c}$ ) for the second compost development (APHA 1998; Jadia and Fulekar 2008).

\section{Experimental Design and Setup}

The study was a pot experiment. Polluted soils were collected and transported from Angwa Magiro and Angwa Kawo of RLGA and the experimental layout was conducted at the biological garden of Federal University of Technology, Minna. The setup was a complete randomized design and the treatments was replicated three times. The experimental pots were filled with $5 \mathrm{~kg}$ polluted soils and the 3 weeks nursed plants for the remediation ( $M$. officinalis $L$ and $S$. acuta) was planted on it. The seed of the two plants selected for this study were collected at the Federal University of Technology, Minna environment in January 2020, they were stored in a dry container until the month of March, 2020 when they were nursed. The nursery took place at the biological garden of Federal University of Technology, Minna. Two beds were prepared and watered for five days before the seeds of both plants were spread on the soil. Each plant seed on the separate bed made. For the seed to sprout, about fifteen litres $(15 \mathrm{~L})$ of water were supply to each of the bed plot where the seed was spread. This was daily and repeatedly done for three weeks. Seedlings of each plants were produced and three seedlings were transplanted to each pot containing the polluted soil for the phytoremediation. Plant growth promoting bacteria (PGPB) were collected as stock culture from Microbiology Laboratory, Federal University of Technology, Minna. The identity of the organism was confirmed as Bacillus safensis which was used for the study. The culture of this organism was prepared in nutrient broth and $100 \mathrm{~mL}$ was applied by spraying on the plant leaves and stems. This was done at three weeks interval until the plants were fully matured. Vermicast $(0.5 \mathrm{~kg})$ was added as fertilizer to the plants, this was done by direct application to the surrounding plants around the root. The application was done twice at two months interval. The set-up was done and monitored for seven (7) months. The physicochemical properties and the microbial counts of the soil were done at a month interval throughout the study period.

\section{Experimental Layout for Phytoremediation Studies}

The experimental design for the phytoremediation is presented in Table 1. The polluted soils were taken from two villages (Angwa Kawo and Angwa Magiro). Two selected plants (Melissa officinalis $L$ and Sida acuta) were used for the remediation. Each plant was subjected to six treatment and they were replicated thrice for each treatment for the two villages. The plants were subjected to $5 \mathrm{~kg}$ soil and were treated as shown in Table 1 .

Table 1: Design of the Phytoremediation Studies 


\begin{tabular}{|c|c|c|}
\hline Treatments & Treatment Code & Details of the Treatment \\
\hline \multirow[t]{14}{*}{ Angwa Kawo } & Melissa officinalis $\mathrm{L}$ & \\
\hline & A & Soil $(5 \mathrm{~kg})+$ M. officinalis L \\
\hline & $\mathrm{B}$ & Soil $(5 \mathrm{~kg})+$ M. officinalis L + PGPB \\
\hline & $\mathrm{C}$ & Soil $(5 \mathrm{~kg})+M$. officinalis L + CDV+ PGPB \\
\hline & $\mathrm{D}$ & Soil $(5 \mathrm{~kg})+M$. officinalis $L+\mathrm{GMV}+\mathrm{PGPB}$ \\
\hline & $\mathrm{E}$ & Soil $(5 \mathrm{~kg})+$ M. officinalis L + CDV \\
\hline & $\mathrm{F}$ & Soil $(5 \mathrm{~kg})+M$. officinalis L + GMV \\
\hline & Sida acuta & \\
\hline & G & Soil $(5 \mathrm{~kg})+$ S. acuta \\
\hline & $\mathrm{H}$ & Soil $(5 \mathrm{~kg})+$ S. acuta + PGPB \\
\hline & 1 & Soil $(5 \mathrm{~kg})+$ S. acuta + CDV+ PGPB \\
\hline & $\mathrm{J}$ & Soil $(5 \mathrm{~kg})+$ S. acuta + GMV+ PGPB \\
\hline & $\mathrm{K}$ & Soil $(5 \mathrm{~kg})+$ S. acuta + CDV \\
\hline & $\mathrm{L}$ & Soil $(5 \mathrm{~kg})+$ S. acuta + GMV \\
\hline \multirow[t]{14}{*}{ Angwa Magiro } & Melissa officinalis $\mathrm{L}$ & \\
\hline & M & Soil $(5 \mathrm{~kg})+$ M. officinalis $L$ \\
\hline & $\mathrm{N}$ & Soil $(5 \mathrm{~kg})+$ M. officinalis L + PGPB \\
\hline & 0 & Soil $(5 \mathrm{~kg})+M$. officinalis L + CDV+ PGPB \\
\hline & $\mathrm{P}$ & Soil $(5 \mathrm{~kg})+M$. officinalis L + GMV+ PGPB \\
\hline & $\mathrm{Q}$ & Soil $(5 \mathrm{~kg})+M$. officinalis L + CDV \\
\hline & $\mathrm{R}$ & Soil $(5 \mathrm{~kg})+M$. officinalis L + GMV \\
\hline & Sida acuta & \\
\hline & $\mathrm{S}$ & Soil $(5 \mathrm{~kg})+S$. acuta \\
\hline & $\mathrm{T}$ & Soil $(5 \mathrm{~kg})+$ S. acuta + PGPB \\
\hline & $U$ & Soil $(5 \mathrm{~kg})+$ S. acuta + CDV+ PGPB \\
\hline & $\mathrm{V}$ & Soil $(5 \mathrm{~kg})+$ S. acuta + GMV+ PGPB \\
\hline & W & Soil $(5 \mathrm{~kg})+$ S. acuta + CDV \\
\hline & $x$ & Soil $(5 \mathrm{~kg})+$ S. acuta + GMV \\
\hline
\end{tabular}

Keys; PGPB= Plant Growth Promoting Bacteria, CDV=Chicken Dropping Vermicompost, GMV= Goat Manure Vermicompost

\section{Determination of Heavy Metal in the Harvested Plants}

After harvesting, plant shoots and roots were separated from soil, carefully washed first with tap water, and then with distilled water until all dirt's were removed. All samples were air-dried the Microbiology laboratory, Federal University of Technology, Minna, for seven days. The samples were oven-dried at $600^{\circ} \mathrm{C}$ until a constant weight were obtained. The dried plant parts were ground to powder using a horizontal grinder (Kai et al. 2012). The dried samples were digested with a mixture (3:1) of concentrated nitric acid and hydrofluoric acid in microwave assisted Kjeldahl digestion. Each microwave extraction vessel was added with $6 \mathrm{~mL}$ of nitric acid and $2 \mathrm{~mL}$ of hydrofluoric acid together with $0.8 \mathrm{~g}$ of plant sample. The vessels were capped and heated in a microwave unit at $800 \mathrm{~W}$ to a temperature of $190^{\circ} \mathrm{C}$ for $20 \mathrm{~min}$ with a pressure of 25 bars. The digested samples were diluted to $50 \mathrm{~mL}$ and subjected to analysis of the metals by atomic absorption spectrophotometer using flame atomization. Results were expressed on dry weight basis of each component (Kai et al. 2012).

\section{Evaluation of Phytoremediation Factors}

i. Bio-concentration factor (BCF): This was calculated using the metal concentration ratio in plant roots to that in soil (Yoon et al. 2006; Nazir et al. 2011) and is given as follows: $\mathrm{BCF}=$ metal concentration in root/metal concentration in soil.

ii. Translocation factor (TF): This is the ratio of metal concentration in plant shoot to that in plant root. TF = metal concentration in plant shoot/metal concentration in plant root (Yoon et al. 2006; Ameh et al. 2019). 
iii. Biological accumulation coefficient (BAC): This is defined as the concentration of metals in plant shoots divided by metal concentration in soil (Nazir et al. 2011) and is given as follows: BAC = concentration of metal in plant shoots/metals concentration in soil.

iv. Enrichment factor (EF): This is the ratio of metal concentration in plant leaves to metal concentration in soil. EF = concentration of metal in leaves/concentration of metal in soil (Lorestani et al. 2011).

\section{XRF Spectroscopy Analysis of Bio Remediated Soil}

Two radioactive isotope sources, cadmium-109 (Cd-109) and americium-241 (Am-241), were used by the NITON XL722S XRF instrument for the production of primary X-rays. Each of these sources emit a specific set of primary X-rays which excite a corresponding range of elements in a sample. When more than one source can excite the element of interest, the appropriate source is selected according to its excitation efficiency for the element of interest. The NITON XL722S instrument was configured with the appropriate sources based on the applications provided with the unit. Soil sample was positioned in front of the source-detector window and sample measurement is initiated. This exposes the sample to primary radiation from the source. Fluorescent and backscattered X-rays from the sample entered through the detector window and are counted by the high-performance, solid-state detector. Elemental concentrations were computed based on ratios of analyte X-ray intensity to source backscatter. The raw ratios are corrected for spectral overlap and inter-element effects using correction coefficients and iteratively computed element concentrations. The NITON XL722S is factory calibrated, and the menu-driven software supports multiple calibrations called "applications." Each application is a complete analysis configuration including elements to be measured, interfering elements in the sample, and a set of calibration coefficients. The AStandard Soil Application@ for the NITON XL722S may was used as: 1) the percentage of the elements of interest are less than $(<) 1.0 \%, 2$ ) the material is of a light matrix (for example, aluminum silicate), and 3 ) elements with atomic number greater than iron do not exceed several percent. Measurement time is user controlled. Shorter measurement times (30 to 60 seconds [s]) are generally used for initial screening and hot spot delineation, while longer measurement times (60 to 300 s) are typically used for higher precision and accuracy requirements.

\section{SEM Analysis}

The soil structural components were captured using scanning electron scanning microscope (SEM: JEOL, USA. Model: JSM-7900F). Soil sample was placed on aluminum holder stub using a double sticky carbon tape, then the sample was completely dried in an oven at $60^{\circ} \mathrm{C}$ for at least 3 hours. "Vent" button located at the display panel of the Microscope table was clicked to release nitrogen into the chamber if there are none after which the sample is placed on the sample holder stubs into the mounting holes and the door was closed gently and EVAC button was pressed. After about 2 minutes of waiting when the sound of rotary pump is heard as the green light is seen on the display and waiting for about 30-45 minutes to achieve high vacuum $<5 \times 10-5$ Pa was observed.

As the vacuum reached proper level, filament and monitor light were $\mathrm{ON}$ and the status of dial positions were checked at the acceleration voltage of $15 \mathrm{KV}$. After the filament was turned on, the red light was shown. With the lowest magnification (10X) selected, Choose TV scan mode was chosen and samples located using trackball. Coarse focus switch was turned on and using the focus knob, working distance was changed to 14 mm. $z$-axis up key was pressed to bring up the sample stage slowly, the screen was looked into to find the z-position where the image is in focus, this was noted down and the coarse focus was turned off. The scanning speed was set to S1 while image set up icon was clicked and mapping option was selected for 1024 normal resolution and frame 1 to close the image set up window. The image acquire icon was switched to record the image. Now the software takes the control over SEM and the monitor was freeze and image saved.

\section{Heavy Metal Removal Efficiency}

The efficiency at which the metal contaminants are removed was determined using (Equation (9) as used by Emenike et al. (2017).

$$
\% \text { of heavy metal removal } \frac{\mathrm{CO}(\mathrm{X})-\mathrm{CF}(\mathrm{X})}{\mathrm{CO}(\mathrm{X})} * 100
$$

Where

$\mathrm{C}_{0}(\mathrm{x})=$ initial concentration of metal, " $\mathrm{x}$ " $=(\mathrm{Pb}, \mathrm{Cd}, \mathrm{As})$ in the soil at the beginning of the experiment.

$C_{F}(x)=$ final concentration of metal, " $x$ " = $(\mathrm{Pb}, \mathrm{Cd}, \mathrm{As})$ in the soil at the end of the experiment. XRF Spectroscopy SEM Analysis of Bio Remediated Soil Were also done by standard methods and result presented

\section{Statistical Analysis}

The collected were assayed and analysed in triplicates using SAS 9.0. All physio-chemical experiments were subjected separately to analysis of variance (ANOVA). Duncan Multiple Range Test (at 5 and 1\%) and Mean \pm SEM were used to test mean comparison for significant effects of the treatment. This was used to identify the significance of differences of the concentration of each element in the soil and plant samples also. SAS version 9.0 was used to analyze the significant effects and means were compared for the treatment, plant parts and location effects with respective Standard Error of means. Canonical discriminant analysis was done with IBM statistics SPSS 20 to determine the combined distribution of the heavy metals as influenced by treatments and assimilated by the plant parts in each location.

\section{Results}

Physicochemical Properties of the Vermicompost 
Table 2: Physicochemical Properties of the Vermi-Composts.

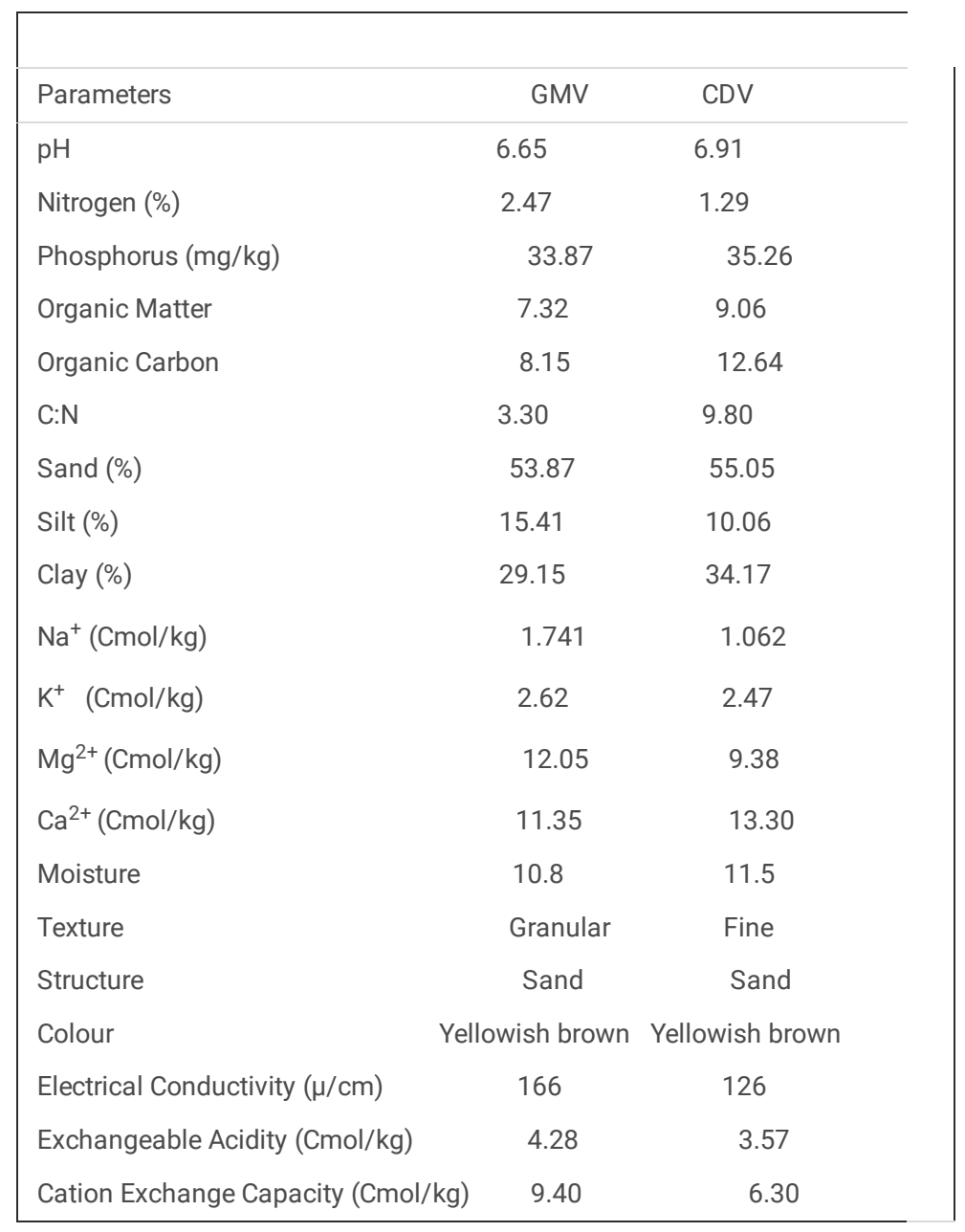

KEY:

GMV- Goat Manure Vermicompost, CDV- Chicken Dropping Vermicompost

$\mathrm{Mg} / \mathrm{kg}$ : milligram per kilogram, $\mathrm{cmol} / \mathrm{kg}$ : centimoles of charge per kilogram

Physicochemical Properties of Soil During the Study.

Mean Square Interactions (ANOVA) of the Physicochemical of the Polluted Soils Across the Study Period

Table 3: Physicochemical Mean Square Interactions of the Polluted Soils Across the Study Period 


\begin{tabular}{|c|c|c|c|c|c|c|c|c|c|c|c|c|}
\hline \multirow[t]{3}{*}{ Sources } & \multirow[t]{3}{*}{ DF } & \multicolumn{11}{|c|}{ Mean Squares } \\
\hline & & $\mathrm{pH}$ & EC & $\% \mathrm{OC}$ & $\% \mathrm{OM}$ & $\% \mathrm{TN}$ & $\mathrm{Ca}$ & $\mathrm{Mg}$ & $\mathrm{Na}$ & K & ExA & C \\
\hline & & & $(\mu / \mathrm{cm})$ & & & & (mg/kg) & $(\mathrm{mg} / \mathrm{kg})$ & $(\mathrm{mg} / \mathrm{kg})$ & (mg/kg) & $\mathrm{Cmol} / \mathrm{kg}$ & C \\
\hline Rep & 5 & $1.85^{\star \star}$ & $43248.13^{\star \star}$ & $7.81^{\star \star}$ & $3.06 * *$ & 0.18 ** & $318096.18^{\star \star}$ & 31378.77 ** & 3565.64 ** & $9699.00 * \star$ & 29.46 & 2 \\
\hline Location & 1 & 0.42 & 361 & 0.08 & $0.84^{\star}$ & 0.10 ** & 7140.25 & 5217.65 & 0.001 & $6408.00 * \star$ & 33.96 & 0 \\
\hline Plant & 1 & $1.15^{\star}$ & 1167.36 & 0.06 & $0.74^{\star}$ & $0.05^{\star *}$ & 25016.69 & 966.17 & 396.67 & 9.10 & 19.16 & 1 \\
\hline Month & 5 & $4.17^{\star \star}$ & $4761.51^{\star \star}$ & $56.56^{\star \star}$ & $3.52^{\star \star}$ & $0.14^{\star *}$ & $286310.45^{\star \star}$ & $24247.44^{\star \star}$ & $1341.17^{\star *}$ & $26794.38^{\star \star}$ & 33.83 & 5 \\
\hline Loc*Plant & 1 & 0.03 & 220.03 & 0.30 & 0.38 & 0.003 & 272.25 & 7909.13* & 3.80 & 89.30 & 25.84 & 0 \\
\hline Loc*Month & 5 & $0.97 \star \star$ & 582.07 & 1.25 & $1.03^{\star \star}$ & 0.005 & $27081.12^{\star}$ & 2019.78 & $321.97 * \star$ & 966.23 & 29.43 & 0 \\
\hline Plant*Month & 5 & 0.50 & 737.19 & 0.77 & $0.43^{*}$ & 0.01 & 3875.83 & 1201.41 & 111.60 & 41.10 & 25.27 & 0 \\
\hline Loc*Plant*Month & 5 & 0.26 & 60.13 & 0.33 & 0.27 & 0.002 & 5702.72 & 2115.07 & 143.81 & 135.83 & 32.12 & 0 \\
\hline Error & 115 & 0.27 & 930.81 & 0.87 & 0.18 & 0.008 & 11466.44 & 1575.42 & 88.94 & 537.10 & 29.02 & 0 \\
\hline R Square (100) & & 56.58 & 69.91 & 77.05 & 68.16 & 67.93 & 71.06 & 63.77 & 73.12 & 75.91 & 19.91 & 7 \\
\hline CV & & 8.02 & 25.06 & 55.20 & 42.81 & 43.23 & 16.53 & 29.16 & 15.70 & 27.00 & 580.64 & 2 \\
\hline Root MSE & & 0.52 & 30.5092 & 0.93 & 0.42 & 0.09 & 107.08 & 39.69 & 9.43 & 23.17 & 5.38 & 0 \\
\hline Mean & & 6.47 & 121.7222 & 1.69 & 0.98 & 0.20 & 647.46 & 136.08 & 60.03 & 85.80 & 0.93 & 3 \\
\hline
\end{tabular}

Key: EC=Electrical Conductivity, OC=Organic Carbon, OM=Organic Matter, Exchangeable Acidity, CEC=Cations Exchange Capacity *Mean Square Values significant at $\mathrm{a}=0.05$; ** Mean Square Values significant at $\mathrm{a}=0.0$

Physicochemical Mean Comparison for the Months of Experiments Across the Parameters Measured

$\mathrm{pH}$ values increased progressively, but the values recorded from May to July were significantly the same, these were lower significantly than pH value in August which is not different from September's value but significantly lower than October's pH value (Table 4).

Table 4: Physicochemical Mean Comparison for the Months of Experiments Across the Parameters Measured.

\begin{tabular}{|c|c|c|c|c|c|c|c|c|c|c|c|}
\hline Months & $\mathrm{pH}$ & $\mathrm{EC} \mu / \mathrm{cm}$ & $\% O C$ & $\% O M$ & $\% \mathrm{TN}$ & Ca mg/kg & $\mathrm{Mg} \mathrm{mg} / \mathrm{kg}$ & $\mathrm{Na} \mathrm{mg/kg}$ & $\mathrm{K} \mathrm{mg/kg}$ & $\begin{array}{l}\text { ExA } \\
\mathrm{Cmol} / \mathrm{kg}\end{array}$ & $\begin{array}{l}\mathrm{CEC} \\
\mathrm{Cmol} / \mathrm{kg}\end{array}$ \\
\hline May & $6.170 c$ & $111.000 \mathrm{~b}$ & $0.462 d$ & $0.664 d$ & $0.118 c$ & $550.000 d$ & $103.830 \mathrm{c}$ & $50.304 c$ & $62.500 c$ & $0.193 a$ & $2.124 \mathrm{e}$ \\
\hline June & $5.981 \mathrm{c}$ & $107.542 b$ & $0.458 d$ & $0.631 \mathrm{~cd}$ & $0.122 c$ & $555.250 \mathrm{~d}$ & $107.630 \mathrm{c}$ & $52.638 c$ & $64.529 c$ & $0.242 a$ & $2.221 \mathrm{e}$ \\
\hline July & $6.160 \mathrm{c}$ & $112.625 b$ & $0.967 \mathrm{~cd}$ & $0.906 \mathrm{c}$ & $0.195 b$ & $585.330 \mathrm{~cd}$ & $131.830 \mathrm{~b}$ & $60.433 b$ & $67.283 c$ & $0.370 a$ & $3.165 d$ \\
\hline August & $6.710 \mathrm{~b}$ & $121.292 \mathrm{ab}$ & $1.313 \mathrm{c}$ & $0.852 \mathrm{~cd}$ & $0.211 \mathrm{~b}$ & $639.580 c$ & $135.410 \mathrm{~b}$ & $62.529 \mathrm{~b}$ & $72.679 c$ & $0.563 a$ & $4.354 c$ \\
\hline September & 6.753ab & $139.292 a$ & $2.493 b$ & $1.194 b$ & $0.264 a$ & $732.500 \mathrm{~b}$ & $146.250 \mathrm{~b}$ & $63.825 b$ & $99.771 b$ & $3.293 a$ & $5.040 \mathrm{~b}$ \\
\hline October & $7.030 \mathrm{a}$ & 138.583a & $4.420 \mathrm{a}$ & $1.646 a$ & $0.307 a$ & $822.080 a$ & 191.580a & $70.488 a$ & $148.063 a$ & $0.905 a$ & $6.011 a$ \\
\hline
\end{tabular}

Down the column values with the same letter are not significantly different from the other.

Key: EC=Electrical Conductivity, OC=Organic Carbon, OM=Organic Matter, Exchangeable Acidity, CEC=Cations Exchange Capacity

Table 5: Physicochemical Mean Separation for pH, Organic Matter and Mg Across the Locations and Months of the Study 


\begin{tabular}{|lllll|}
\hline Location & Month & \multicolumn{3}{l|}{ Mean \pm Std. Error Mean } \\
& & pH & $\%$ OM & Mg mg/kg \\
\hline Ang K. & August & $7.007 \pm 0.176 \mathrm{ab}$ & $0.810 \pm 0.079 \mathrm{~cd}$ & $122.183 \pm 12.456 \mathrm{de}$ \\
\hline Ang K. & July & $6.164 \pm 0.187 \mathrm{e}$ & $1.012 \pm 0.139 \mathrm{bc}$ & $111.017 \pm 11.744 \mathrm{de}$ \\
\hline Ang K. & June & $5.746 \pm 0.215 \mathrm{f}$ & $0.543 \pm 0.064 \mathrm{e}$ & $109.825 \pm 8.920 \mathrm{de}$ \\
\hline Ang K. & May & $6.092 \pm 0.166 \mathrm{ef}$ & $0.561 \pm 0.060 \mathrm{e}$ & $105.767 \pm 8.999 \mathrm{e}$ \\
\hline Ang K. & October & $7.273 \pm 0.090 \mathrm{a}$ & $2.092 \pm 0.376 \mathrm{a}$ & $189.517 \pm 28.135 \mathrm{ab}$ \\
\hline Ang K. & Sept. & $6.842 \pm 0.165 \mathrm{bc}$ & $1.334 \pm 0.239 \mathrm{~b}$ & $142.100 \pm 15.966 \mathrm{~cd}$ \\
\hline Ang M. & August & $6.414 \pm 0.209 \mathrm{de}$ & $0.894 \pm 0.120 \mathrm{~cd}$ & $148.642 \pm 14.984 \mathrm{bcd}$ \\
\hline Ang M. & July & $6.157 \pm 0.172 \mathrm{e}$ & $0.801 \pm 0.093 \mathrm{~cd}$ & $152.642 \pm 14.569 \mathrm{bc}$ \\
\hline Ang M. & June & $6.217 \pm 0.155 \mathrm{e}$ & $0.718 \pm 0.066 \mathrm{~d}$ & $105.425 \pm 11.352 \mathrm{e}$ \\
\hline Ang M. & May & $6.248 \pm 0.172 \mathrm{e}$ & $0.768 \pm 0.072 \mathrm{~d}$ & $101.900 \pm 10.720 \mathrm{e}$ \\
\hline Ang M. & October & $6.774 \pm 0.129 \mathrm{c}$ & $1.201 \pm 0.150 \mathrm{~b}$ & $193.642 \pm 18.758 \mathrm{a}$ \\
\hline Ang M. & September & $6.664 \pm 0.155 \mathrm{~cd}$ & $1.053 \pm 0.138 \mathrm{bc}$ & $150.392 \pm 14.888 \mathrm{bc}$ \\
\hline
\end{tabular}

\section{ANOVA, Data represent means \pm SEM}

Considering the two locations AK and AM across the months for this study, the mean separation for the $\mathrm{pH}, \mathrm{OM}$ and $\mathrm{Mg}$ location was higher (7.273, 2.092 and 189.517 respectively) in the of October while the lowest for $\mathrm{pH}$ and OM $(5.746,0.543$ respectively) were recorded in the month of June for $\mathrm{AK}$ location. These values also showed significant differences from other values from the other months (Table 5). It has been reported that the organic matter of the soil is one of the key factors determining the plant capacity to take up metals from the soil (Nejad et al. 2017). With complexes formed by organic matter and heavy metals, its content of OM may affect the specificity of heavy metals in soil (Koretsky 2000). In line with this principle, high OM content was reported to decrease Cd and $\mathrm{Ni}$ in soil solution (et al. 2007). The application of vermicast as a source of OM is a well-known practice to improve soil properties (e.g., enrichment of humic acids molecules in carbon and nitrogen) because of its sufficiently slow mineralization (et al. 2005).

\section{XRF Spectrum Analysis of the Remediated Soil}

The $x$-ray fluorescence spectroscopy of the remediated soil is presented in Figures 3 and 4 . The reduction in peaks indicated that the remediation processes have taken place after seven months.

Table 6: Mean Value of $\mathrm{Cd}, \mathrm{As}, \mathrm{Pb}$ and Their Interactions Between Plant Parts and the Two Location.

\begin{tabular}{|llll|}
\hline Location & $\mathrm{Cd}$ & As & $\mathrm{Pb}$ \\
\hline & Root & & \\
\hline Angwan Kawo & $0.141 \pm 0.013 \mathrm{~b}$ & $1.873 \pm 0.103 \mathrm{e}$ & $5.491 \pm 0.388 \mathrm{ef}$ \\
\hline Angwan Magiro & $0.163 \pm 0.014 \mathrm{~b}$ & $2.934 \pm 0.200 \mathrm{bc}$ & $7.434 \pm 0.590 \mathrm{~cd}$ \\
\hline & Stem & & \\
\hline Angwan Kawo & $0.215 \pm 0.019 \mathrm{a}$ & $2.616 \pm 0.197 \mathrm{~cd}$ & $6.518 \pm 0.573 \mathrm{de}$ \\
\hline Angwan Magiro & $0.202 \pm 0.019 \mathrm{ab}$ & $3.353 \pm 0.221 \mathrm{~b}$ & $7.653 \pm 0.643 \mathrm{c}$ \\
\hline & Leaf & & \\
\hline Angwan Kawo & $0.180 \pm 0.017 \mathrm{ab}$ & $2.015 \pm 0.169 \mathrm{~d}$ & $5.599 \pm 0.492 \mathrm{def}$ \\
\hline Angwan Magiro & $0.140 \pm 0.012 \mathrm{~b}$ & $2.743 \pm 0.173 \mathrm{c}$ & $6.599 \pm 0.549 \mathrm{~d}$ \\
\hline & Seed & & \\
\hline Angwan Kawo & $0.153 \pm 0.017 \mathrm{~b}$ & $1.384 \pm 0.162 \mathrm{f}$ & $5.108 \pm 0.501 \mathrm{f}$ \\
\hline Angwan Magiro & $0.133 \pm 0.017 \mathrm{~b}$ & $2.339 \pm 0.197 \mathrm{~cd}$ & $6.149 \pm 0.493 \mathrm{de}$ \\
\hline & Soil & & \\
\hline Angwan Kawo & $0.160 \pm 0.030 \mathrm{~b}$ & $2.065 \pm 0.350 \mathrm{~d}$ & $9.116 \pm 0.388 \mathrm{~b}$ \\
\hline Angwan Magiro & $0.136 \pm 0.020 \mathrm{~b}$ & $5.856 \pm 0.571 \mathrm{a}$ & $17.501 \pm 1.091 \mathrm{a}$ \\
\hline
\end{tabular}


Table 7: Mean of $\mathrm{Cd}, \mathrm{As}, \mathrm{Pb}$ Value with the Treatments and Locations Interaction.

\begin{tabular}{|c|c|c|c|}
\hline Locations & Cd & As & $\mathrm{Pb}$ \\
\hline & \multicolumn{3}{|c|}{ Soil $(5 \mathrm{~kg})+M$. officinalis $\mathrm{L}$. } \\
\hline Angwan Kawo & $0.159 \pm 0.059 a b$ & $1.961 \pm 0.503 \mathrm{~cd}$ & $3.729 \pm 1.222 \mathrm{e}$ \\
\hline \multirow[t]{2}{*}{ Angwan Magiro } & $0.113 \pm 0.030 \mathrm{~b}$ & $3.030 \pm 0.996 b$ & $5.743 \pm 1.810 \mathrm{~cd}$ \\
\hline & \multicolumn{3}{|c|}{ Soil $(5 \mathrm{~kg})+M$. officinalis L + PGPB } \\
\hline Angwan Kawo & $0.141 \pm 0.042 a b$ & $1.379 \pm 0.319 d$ & $4.306 \pm 0.847 \mathrm{e}$ \\
\hline \multirow[t]{2}{*}{ Angwan Magiro } & $0.143 \pm 0.022 a b$ & $3.747 \pm 0.773 \mathrm{ab}$ & $7.135 \pm 1.784 \mathrm{c}$ \\
\hline & \multicolumn{3}{|c|}{ Soil $(5 \mathrm{~kg})+M$. officinalis L + CDV+ PGPB } \\
\hline Angwan Kawo & $0.193 \pm 0.016 a$ & $2.318 \pm 0.374 \mathrm{bc}$ & $7.819 \pm 0.392 b c$ \\
\hline \multirow[t]{2}{*}{ Angwan Magiro } & $0.174 \pm 0.032 a b$ & $3.878 \pm 0.193 a$ & $11.009 \pm 0.443 a$ \\
\hline & \multicolumn{3}{|c|}{ Soil $(5 \mathrm{~kg})+$ M. officinalis L + GMV+ PGPB } \\
\hline Angwan Kawo & $0.192 \pm 0.022 a$ & $1.985 \pm 0.316 \mathrm{~cd}$ & $8.303 \pm 0.440 \mathrm{bc}$ \\
\hline \multirow[t]{2}{*}{ Angwan Magiro } & $0.181 \pm 0.042 \mathrm{ab}$ & $3.329 \pm 0.357 b$ & $10.793 \pm 0.513 a$ \\
\hline & \multicolumn{3}{|c|}{ Soil $(5 \mathrm{~kg})+M$. officinalis $L+C D V$} \\
\hline Angwan Kawo & $0.193 \pm 0.005 a$ & $2.147 \pm 0.119 \mathrm{~cd}$ & $7.111 \pm 0.602 c$ \\
\hline \multirow[t]{2}{*}{ Angwan Magiro } & $0.168 \pm 0.035 \mathrm{ab}$ & $2.729 \pm 0.224 b c$ & $10.054 \pm 0.997 a$ \\
\hline & \multicolumn{3}{|c|}{ Soil $(5 \mathrm{~kg})+M$. officinalis $L+\mathrm{GMV}$} \\
\hline Angwan Kawo & $0.193 \pm 0.024 a$ & $2.192 \pm 0.259 \mathrm{~cd}$ & $7.255 \pm 0.520 b c$ \\
\hline \multirow[t]{2}{*}{ Angwan Magiro } & $0.115 \pm 0.021 b$ & $2.935 \pm 0.408 \mathrm{bc}$ & $10.312 \pm 0.480 a$ \\
\hline & \multicolumn{3}{|c|}{ Soil $(5 \mathrm{~kg})+$ S. acuta } \\
\hline Angwan Kawo & $0.059 \pm 0.025 b$ & $1.791 \pm 0.490 \mathrm{~cd}$ & $3.574 \pm 0.886 \mathrm{e}$ \\
\hline \multirow[t]{2}{*}{ Angwan Magiro } & $0.142 \pm 0.029 \mathrm{ab}$ & $3.072 \pm 1.100 \mathrm{~b}$ & $7.732 \pm 2.464 \mathrm{bc}$ \\
\hline & \multicolumn{3}{|c|}{ Soil $(5 \mathrm{~kg})+$ S. acuta + PGPB } \\
\hline Angwan Kawo & $0.190 \pm 0.030 \mathrm{ab}$ & $1.971 \pm 0.352 \mathrm{~cd}$ & $4.379 \pm 0.561 \mathrm{de}$ \\
\hline \multirow[t]{2}{*}{ Angwan Magiro } & $0.129 \pm 0.013 a b$ & $3.805 \pm 0.769 a$ & $6.899 \pm 2.583 c$ \\
\hline & \multicolumn{3}{|c|}{ Soil $(5 \mathrm{~kg})+$ S. acuta + CDV+ PGPB } \\
\hline Angwan Kawo & $0.197 \pm 0.025 a$ & $2.144 \pm 0.315 \mathrm{~cd}$ & $8.365 \pm 0.379 b c$ \\
\hline \multirow[t]{2}{*}{ Angwan Magiro } & $0.173 \pm 0.015 \mathrm{ab}$ & $4.271 \pm 0.227 a$ & $9.006 \pm 0.398 b$ \\
\hline & \multicolumn{3}{|c|}{ Soil $(5 \mathrm{~kg})+$ S. acuta + GMV+ PGPB } \\
\hline Angwan Kawo & $0.195 \pm 0.035 a$ & $2.153 \pm 0.329 \mathrm{~cd}$ & $8.616 \pm 0.492 b c$ \\
\hline \multirow[t]{2}{*}{ Angwan Magiro } & $0.175 \pm 0.026 a b$ & $3.911 \pm 0.309 a$ & $10.261 \pm 0.585 a$ \\
\hline & \multicolumn{3}{|c|}{ Soil $(5 \mathrm{~kg})+S$. acuta + CDV } \\
\hline Angwan Kawo & $0.148 \pm 0.024 a b$ & $1.953 \pm 0.215 \mathrm{~cd}$ & $6.117 \pm 0.933 \mathrm{~cd}$ \\
\hline \multirow[t]{2}{*}{ Angwan Magiro } & $0.171 \pm 0.013 a b$ & $3.117 \pm 0.306 b$ & $9.901 \pm 2.071 \mathrm{a}$ \\
\hline & \multicolumn{3}{|c|}{ Soil $(5 \mathrm{~kg})+$ S. acuta + GMV } \\
\hline Angwan Kawo & $0.178 \pm 0.025 a b$ & $1.895 \pm 0.387 \mathrm{~cd}$ & $6.825 \pm 0.411 \mathrm{c}$ \\
\hline Angwan Magiro & $0.173 \pm 0.024 a b$ & $3.518 \pm 0.215 \mathrm{ab}$ & $9.961 \pm 1.553 a$ \\
\hline
\end{tabular}

ANOVA, Data represent means \pm SEM

Accumulation and Translocation of Metals in the Plants Used for the Study

Table 8: Accumulation and Translocation of $\mathrm{Cd}, \mathrm{As}$, and $\mathrm{Pb}$ in $\mathrm{M}$. officinalis $\mathrm{L}$ used for remediation of Angwan Kawo Soil 


\begin{tabular}{|c|c|c|c|c|c|}
\hline Treatments & Metals & $\mathrm{BCF}$ & TF & BAC & EF \\
\hline \multirow[t]{3}{*}{ Soil $(5 \mathrm{~kg})+$ M. officinalis $L$} & $\mathrm{Cd}$ & 0.17 & 1.59 & 0.27 & 0.10 \\
\hline & As & 0.25 & 2.11 & 0.5 & 0.20 \\
\hline & $\mathrm{Pb}$ & 0.33 & 0.56 & 0.18 & 0.06 \\
\hline \multirow[t]{3}{*}{ Soil $(5 \mathrm{~kg})+M$. officinalis $L+P G P B$} & $\mathrm{Cd}$ & 0.09 & 5.68 & 0.53 & 0.23 \\
\hline & As & 0.23 & 3.36 & 0.79 & 0.27 \\
\hline & $\mathrm{Pb}$ & 0.22 & 3.71 & 0.83 & 0.28 \\
\hline \multirow[t]{3}{*}{ Soil $(5 \mathrm{~kg})+M$. officinalis $L+C D V+$ PGPB } & $\mathrm{Cd}$ & 1.25 & 5.33 & 6.66 & 2.25 \\
\hline & As & 6.0 & 4.86 & 29.22 & 7.72 \\
\hline & $\mathrm{Pb}$ & 1.13 & 3.97 & 4.51 & 1.54 \\
\hline \multirow[t]{3}{*}{ Soil $(5 \mathrm{~kg})+M$. officinalis $L+\mathrm{GMV}+\mathrm{PGPB}$} & $\mathrm{Cd}$ & 4.18 & 4.0 & 16.74 & 0.32 \\
\hline & As & 5.38 & 4.22 & 22.70 & 5.70 \\
\hline & $\mathrm{Pb}$ & 1.16 & 3.77 & 4.41 & 1.50 \\
\hline \multirow[t]{3}{*}{ Soil $(5 \mathrm{~kg})+M$. officinalis $L+C D V$} & $\mathrm{Cd}$ & 0.90 & 2.95 & 2.61 & 0.90 \\
\hline & AS & 0.82 & 2.83 & 2.32 & 0.79 \\
\hline & $\mathrm{Pb}$ & 0.53 & 3.22 & 1.69 & 0.56 \\
\hline \multirow[t]{3}{*}{ Soil $(5 \mathrm{~kg})+M$. officinalis $L+\mathrm{GMV}$} & $\mathrm{Cd}$ & 8.46 & 3.27 & 27.69 & 10.76 \\
\hline & AS & 4.32 & 3.93 & 17.02 & 6.95 \\
\hline & $\mathrm{Pb}$ & 0.54 & 3.61 & 1.93 & 0.64 \\
\hline
\end{tabular}

Key: BCF (Bio-Concentration Factor) = metal concentration ratio of plant roots to soil, TF (Translocation Factor) = metal concentration ratio of plant shoots to roots, BAC (Biological Accumulation Coefficient) $=$ metal concentration ratio of plant shoots to soil, EF (Enrichment Factor) $=$ metal concentration ratio of plant leaves to soil. Values> 1 are in bold font

Table 9: Accumulation and Translocation of $\mathrm{Cd}, \mathrm{As}$, and $\mathrm{Pb}$ in $\mathrm{S}$. acuta used for Remediation of Angwan Kawo Soil

\begin{tabular}{|c|c|c|c|c|c|}
\hline Treatments & Metals & $\mathrm{BCF}$ & TF & BAC & EF \\
\hline \multirow[t]{3}{*}{ Soil $(5 \mathrm{~kg})+$ S. acuta } & $\mathrm{Cd}$ & 0.04 & 3.5 & 0.15 & 0.08 \\
\hline & As & 0.25 & 1.71 & 0.43 & 0.19 \\
\hline & $\mathrm{Pb}$ & 0.24 & 2.18 & 0.53 & 0.18 \\
\hline \multirow[t]{3}{*}{ Soil $(5 \mathrm{~kg})+$ S. acuta + PGPB } & $\mathrm{Cd}$ & 5.21 & 5.91 & 30.86 & 12.17 \\
\hline & As & 0.30 & 2.88 & 0.87 & 0.30 \\
\hline & $\mathrm{Pb}$ & 0.43 & 2.69 & 1.18 & 0.43 \\
\hline \multirow[t]{3}{*}{ Soil $(5 \mathrm{~kg})+$ S. acuta + CDV+ PGPB } & $\mathrm{Cd}$ & 5.25 & 3.47 & 18.25 & 4.75 \\
\hline & As & 5.54 & 4.04 & 22.40 & 9.0 \\
\hline & $\mathrm{Pb}$ & 1.08 & 3.19 & 3.45 & 1.03 \\
\hline \multirow[t]{3}{*}{ Soil $(5 \mathrm{~kg})+$ S. acuta $+\mathrm{GMV}+\mathrm{PGPB}$} & $\mathrm{Cd}$ & 4.25 & 4.47 & 19.0 & 4.0 \\
\hline & As & 34.14 & 3.46 & 18.42 & 33.85 \\
\hline & $\mathrm{Pb}$ & 1.44 & 2.72 & 3.92 & 1.20 \\
\hline \multirow[t]{3}{*}{ Soil $(5 \mathrm{~kg})+$ S. acuta + CDV } & $\mathrm{Cd}$ & 1.45 & 3.57 & 5.20 & 1.04 \\
\hline & AS & 1.31 & 2.37 & 3.50 & 0.73 \\
\hline & $\mathrm{Pb}$ & 0.39 & 3.03 & 1.20 & 0.35 \\
\hline \multirow[t]{3}{*}{ Soil $(5 \mathrm{~kg})+$ S. acuta + GMV } & $\mathrm{Cd}$ & 3.0 & 4.53 & 13.6 & 4.40 \\
\hline & AS & 13.5 & 2.42 & 32.8 & 14.90 \\
\hline & $\mathrm{Pb}$ & 0.66 & 3.75 & 2.47 & 0.79 \\
\hline
\end{tabular}


Key: BCF (Bio-Concentration Factor) = metal concentration ratio of plant roots to soil, TF (Translocation Factor) = metal concentration ratio of plant shoots to roots, BAC (Biological Accumulation Coefficient) $=$ metal concentration ratio of plant shoots to soil, EF (Enrichment Factor) $=$ metal concentration ratio of plant leaves to soil. Values> 1 are in bold font

Table 10: Accumulation and Translocation of $\mathrm{Cd}, \mathrm{As}$, and $\mathrm{Pb}$ in $\mathrm{M}$. officinalis L Used for Remediation of Angwan Magiro Soil.

\begin{tabular}{|c|c|c|c|c|c|}
\hline Treatments & Metals & BCF & TF & BAC & EF \\
\hline \multirow[t]{3}{*}{ Soil $(5 \mathrm{~kg})+$ M. officinalis L } & $\mathrm{Cd}$ & 0.34 & 1.56 & 0.54 & 0.10 \\
\hline & As & 0.11 & 3.06 & 0.34 & 0.17 \\
\hline & $\mathrm{Pb}$ & 0.15 & 2.41 & 0.36 & 0.10 \\
\hline \multirow[t]{3}{*}{ Soil $(5 \mathrm{~kg})+$ M. officinalis $L+P G P B$} & $\mathrm{Cd}$ & 0.16 & 10.5 & 1.75 & 0.54 \\
\hline & As & 0.27 & 2.82 & 0.77 & 0.23 \\
\hline & $\mathrm{Pb}$ & 0.22 & 2.53 & 0.55 & 0.17 \\
\hline \multirow[t]{3}{*}{ Soil $(5 \mathrm{~kg})+M$. officinalis $L+C D V+P G P B$} & $\mathrm{Cd}$ & 12.5 & 2.44 & 30.5 & 7.50 \\
\hline & As & 1.42 & 3.11 & 4.42 & 1.49 \\
\hline & $\mathrm{Pb}$ & 0.85 & 2.89 & 2.46 & 0.88 \\
\hline \multirow[t]{3}{*}{ Soil $(5 \mathrm{~kg})+M$. officinalis $L+\mathrm{GMV}+\mathrm{PGPB}$} & $\mathrm{Cd}$ & 18.13 & 2.03 & 36.88 & 5.63 \\
\hline & As & 4.15 & 2.65 & 11.0 & 3.97 \\
\hline & $\mathrm{Pb}$ & 1.48 & 2.97 & 4.40 & 1.37 \\
\hline \multirow[t]{3}{*}{ Soil $(5 \mathrm{~kg})+M$. officinalis L + CDV } & $\mathrm{Cd}$ & 1.58 & 2.74 & 4.33 & 1.5 \\
\hline & AS & 2.25 & 2.94 & 6.63 & 2.26 \\
\hline & $\mathrm{Pb}$ & 0.51 & 2.89 & 1.48 & 0.49 \\
\hline \multirow[t]{3}{*}{ Soil $(5 \mathrm{~kg})+M$. officinalis $L+\mathrm{GMV}$} & $\mathrm{Cd}$ & 0.5 & 2.60 & 1.3 & 0.55 \\
\hline & AS & 0.43 & 2.97 & 1.28 & 0.41 \\
\hline & $\mathrm{Pb}$ & 0.70 & 3.11 & 2.4 & 0.78 \\
\hline
\end{tabular}

Key: BCF (Bio-Concentration Factor) = metal concentration ratio of plant roots to soil, TF (Translocation Factor) = metal concentration ratio of plant shoots to roots, $\mathrm{BAC}$ (Biological Accumulation Coefficient) $=$ metal concentration ratio of plant shoots to soil, EF (Enrichment Factor) $=$ metal concentration ratio of plant leaves to soil. Values> 1 are in bold font

Table 11: Accumulation and Translocation of $\mathrm{Cd}, \mathrm{As}$, and $\mathrm{Pb}$ in $\mathrm{S}$. acuta L Used for Remediation of Angwan Magiro Soil 


\begin{tabular}{|c|c|c|c|c|c|}
\hline Treatments & Metals & BCF & TF & BAC & $\mathrm{EF}$ \\
\hline \multirow[t]{3}{*}{ Soil $(5 \mathrm{~kg})+$ S. acuta } & $\mathrm{Cd}$ & 1.94 & 0.44 & 0.85 & 0.17 \\
\hline & As & 0.14 & 1.59 & 0.23 & 0.06 \\
\hline & $\mathrm{Pb}$ & 0.13 & 2.57 & 0.35 & 0.12 \\
\hline \multirow[t]{3}{*}{ Soil $(5 \mathrm{~kg})+$ S. acuta + PGPB } & $\mathrm{Cd}$ & 1.75 & 2.93 & 5.13 & 1.38 \\
\hline & As & 0.19 & 4.24 & 0.83 & 0.25 \\
\hline & $\mathrm{Pb}$ & 0.08 & 3.08 & 0.25 & 0.11 \\
\hline \multirow[t]{3}{*}{ Soil $(5 \mathrm{~kg})+$ S. acuta + CDV+ PGPB } & $\mathrm{Cd}$ & 1.15 & 3.86 & 4.46 & 1.07 \\
\hline & As & 0.76 & 2.78 & 2.14 & 0.66 \\
\hline & $\mathrm{Pb}$ & 0.92 & 2.53 & 2.32 & 0.73 \\
\hline \multirow{3}{*}{ Soil $(5 \mathrm{~kg})+$ S. acuta $+\mathrm{GMV}+\mathrm{PGPB}$} & $\mathrm{Cd}$ & 10.37 & 4.09 & 42.5 & 13.13 \\
\hline & As & 0.85 & 2.38 & 2.02 & 0.64 \\
\hline & $\mathrm{Pb}$ & 0.93 & 2.37 & 2.19 & 0.72 \\
\hline \multirow[t]{3}{*}{ Soil $(5 \mathrm{~kg})+$ S. acuta + CDV } & $\mathrm{Cd}$ & 0.95 & 2.43 & 4.05 & 1.79 \\
\hline & AS & 0.55 & 3.56 & 1.96 & 0.67 \\
\hline & $\mathrm{Pb}$ & 0.26 & 2.74 & 0.72 & 0.20 \\
\hline \multirow[t]{3}{*}{ Soil $(5 \mathrm{~kg})+$ S. acuta + GMV } & $\mathrm{Cd}$ & 1.0 & 2.63 & 2.63 & 0.96 \\
\hline & AS & 0.73 & 2.86 & 2.09 & 0.11 \\
\hline & $\mathrm{Pb}$ & 0.37 & 2.64 & 0.98 & 0.29 \\
\hline
\end{tabular}

Key: BCF (Bio-Concentration Factor) = metal concentration ratio of plant roots to soil, TF (Translocation Factor) $=$ metal concentration ratio of plant shoots to roots, BAC (Biological Accumulation Coefficient) $=$ metal concentration ratio of plant shoots to soil, EF (Enrichment Factor) $=$ metal concentration ratio of plant leaves to soil. Values> 1 are in bold font

\section{Heavy Metal Removal Efficiency}

The efficiency at which the metal contaminants were removed was determined (Figures 7-10).

\section{Canonical Discriminant Analysis of Heavy metal Dispersion Across the Treatments and Plants Parts}

\section{Discussion}

Soil structure quality are decreased due to toxic accumulation of heavy metals and this in turn affects the general ecosystem which do not support plants growth and also disturbing the activities of soil microbes (Daia et al. 2004; Sunitha et al. 2014). Immobilization of heavy metals (for example, Pb, Ni, Cd, As, Co) is made possible by organic matter amendments for soil enrichment (Basta and McGowen 2004) and also increases the plants growth rates in phytoremediation process which could lead to an increase in the plants removal efficacy (Wang et al. 2012). Vermicompost processes is quite different from the traditional ways of composting which requires a thermophilic phase, while vermicompost undertakes a mesophilic change. Vermicompost consist of fine texture when produced, it also has a peat-like material and this reinforce its structural characteristics in water retention and facilitates aeration (Belliturk et al. 2015). This as well causes increase in the cation exchange capacity (CEC) of the soils, promoting adsorption of positive ions, including heavy metals (Herwijnen et al. 2007).

\section{$\mathrm{pH}$}

The results of physicochemical parameters of GMV and CDV vermicompost are represented in Table 2 after 60 days of vermicomposting process. The CDV had a slightly higher $\mathrm{pH}$ (6.91) than GMV (6.65) after 60 days (Table 2). Earlier research studies reported similar results during vermicomposting of different wastes (Garg and Gupta 2011; Mousavi et al. 2019). Nitrogen and phosphorus mineralization into nitrites/nitrates and orthophosphates could be the reason why $\mathrm{pH}$ decreases and transformation of organic waste into organic acids (Ndegwa et al. 2000).

\section{Electrical conductivity (EC)}

Results of measuring samples characteristics showed that in the two set up, the EC was higher in GMV (166 $\mu / \mathrm{cm})$ than CDV (126 $\mu / \mathrm{cm})($ Table 2$)$. According to the results of previous studies decomposition of organic material and release of minerals in the form of cations during vermicomposting may have increase EC (Tognetti et al. 2005; Khwairakpam and Bhargava 2009).

\section{Organic Carbon (OC)}


The organic carbon content followed a trend of CDV (12.64) which was higher than the GMV (8.15) (Table 2). Some researchers have stated that earthworms adjust the mixture condition of the feed, by so doing, it aids the carbon losses from the feed mixture by microbial respiration in the form of $\mathrm{CO}_{2}$ (Elvira et al. 1996; Aira et al. 2007; Hait and Tare 2011). Wani et al. (2013) investigated vermicomposting of wastes from garden, cow dung and wastes from kitchen using earthworm Eisenia fetida. Their findings affirmed the OC reduction at the end of the study. Sharma (2003) also observed that a large fraction of OC can be broken down into $\mathrm{CO}_{2}$ during vermicomposting process of municipal solid waste. Nutrient composition that are commonly available in vermicompost is as follows: N 0.5-1.50\%, P 0.1-0.30\%, Ca and Mg 22.67-47.60 meq/100 g, OC 9.5-17.98\%, K 0.15-0.56\%, Na 0.06-0.30\%, Zn 5.70-11.50 mg/kg, Sulfur 128$548 \mathrm{mg} / \mathrm{kg}$, Cu 2-9.50 mg/kg, Fe 2-9.30 mg/kg, (Mousavi et al. 2019). Vermicomposting however, allows biological transformation of wastes into a valued organic fertilizer.

\section{Nitrogen}

The content of nitrogen exhibited a noticeable difference when comparing CDV and GMV. The GMV had more nitrogen content $2.47 \%$ than its content in CDV $1.29 \%$ after 60 days of the vermicomposting process (Table 2). Mousavi et al. (2019) reported that during vermicomposting of tomato-fruit wastes, concentrations of nitrogen progressively increased by 35\%, after 150 day (Fernández-Gómez et al. 2010). The differences observed in nitrogen content could be due to mineralization of C-rich matters in GMV and the action of $\mathrm{N}$ - fixing bacteria that are presented in the feed mixtures (Plaza et al. 2008). Suthar et al. (2006) also reported that earthworms can add nitrogen in the form of mucus, growth promoting hormones and enzymes during digestion of organic waste. Available results of study by Mousavi et al. (2019) showed that nitrogen content of kitchen waste compost, rotting foliage and cow dung gave $2.16 \%$-fold in comparison to initial waste mixtures. This result is similar to the value obtained by this study which could be as a result of GMV have similar content with the cow dung used by the researcher. Studies have also shown that vermicomposting causes a significant increase in the total nitrogen content after the activity of worms (Garg and Gupta 2011; Soobhany et al. 2015).

\section{C/N ratio}

Considering the carbon/nitrogen ratio, which is one of the main convectional indicators of the compost maturation (Mousavi et al. 2019), is the stabilization and mineralization of organic wastes during tte process of vermicompost. The carbon/nitrogen ratio of CDV had higher value of 9.80 to 3.30 of GMV. These represented the result obtained in the organic carbon and nitrogen content of the research. This modification may be due to change in the $\mathrm{OC}$ and $\mathrm{N}$ relative concentration as showed in Table 2. This was consistent by the findings of Kaur et al. (2010) that the Carbon/Nitrogen ratio decreased due to a higher loss of carbon accompanied. To show compost maturation, studies also showed that C:N ratio is one of the main indicators used (Malafaia et al. 2015).

\section{Phosphorous}

As recorded, the concentration of phosphorus in the vermicompost had related value of 33.87 (GMV) and 35.26 (CDV). It could be that processing time, raw materials, materials digested by worms, the species of the worms, and the condition of the test are the reasons for this value (Ndegwa et al. 2000). The passage of organic matter through the intestines of earthworms may be a reason to add some phosphorous to the worm shed, which may consequently increase the available phosphorus to plants, which may be the cause of the increase in phosphorus content for the treatments in this study. It has also been reported that during the decomposition of organic material, microorganisms produce acid that solubilizes insoluble phosphorus and subsequently causes an increase in the phosphorus concentration of vermicompost (Vig et al. 2011).

\section{Macro-nutrient ( $\mathrm{Na}, \mathrm{K}, \mathrm{Mg}$ and $\mathrm{Ca}$ )}

The macro nutrient ( $\mathrm{Na}, \mathrm{K}, \mathrm{Mg}$ and $\mathrm{Ca}$ ) content of the two vermicast GMV and CDV had 1.741, 2.62, 12.05, 11.35 and 1.062, 2.47, 9.38 13.30 respectively. of all treatments Ca showed the highest value for both GMV and CDV $(11.35,13.30)$ (Table 2). Yadav and Garg (2011) revealed similar outcome in the vermicomposting of mixed feed using cow dung, poultry droppings, and food industry sludge using Eisenia fetida.

Table 3 (ANOVA table) shows that the two locations had significant effect on organic matter (at $p<0.05$ ), total carbon and potassium (at $p<0.01$ ), while the two plants significantly affected the content of $\mathrm{pH}$, organic matter (at $\mathrm{p}<0.05)$ and total nitrogen $(\mathrm{at} p<0.01)$. Meanwhile the months (duration of the experiment) had significant effect on all the parameters at $p<0.01$ except exchangeable acidity. Location-plant interactions had significant effect on $\mathrm{Mg}$ only (at $\mathrm{p}<0.05$ ); Location-Month interactions were significant on $\mathrm{pH}, \mathrm{OM}$ and $\mathrm{Na}$ contents (at $\mathrm{p}<0.01$ ), also on $\mathrm{Ca}$ (at $\mathrm{p}<0.05$ ), while Plant-Month interaction was only significant on OM (at $\mathrm{p}<0.05)($ Table 3$)$

The phytoavailability of heavy metals are influenced by the $\mathrm{pH}$ of the soil while the ability to move in the soil solution and heavy metal solubility are influenced by soil acidity (Elekes 2014). The most mobile under acidic conditions is the metal cations while oxide minerals in this $\mathrm{pH}$ range are sorb by anions (Dzombak and Morel 1987). This follow a trend that as more metals are released into the soil solution due to competition with $\mathrm{H}^{+}$ions at low $\mathrm{pH}$, heavy metal bioavailability increases (Dinev et al. 2008). But when $\mathrm{pH}$ value is high, precipitation of cations to minerals occurred causing metal anions to mobilized (Takac, et al. 2009). Many of the heavy metals in the soil may not be available to plants, especially $\mathrm{Cr}$ and $\mathrm{Pb}$ are intrinsically immobile when the pH is at neutral or alkaline level. The role controlling the behavior of metals in the soil are determined by soil organic matter as reported by (Singh and Kalamdhad 2013). Organic carbon presence surges the cation exchange capacity of the soil which holds nutrients integrated by plants (Yobouet et al. 2010). With the pH of 7.03 in October which was the highest and significantly different from other, it shows that metal mobility was lower than the $\mathrm{pH}$ value of other months (Table 4). the significant differences for all the parameters followed a common patter and it shows that obtained values for the month of October were significantly different from other months. These could be as a result of the phytoremediation process getting to the peak and all nutrients declined. Edaphic factor of the soil and the available nutrients determines the metal uptakes by the plants and plant species (Afonne and Ifediba 2020; Maddela et al. 2020). Another measure used to ascertain the uptake of metals by plants is the soil-to-plant transfer factors. This factor will however help to develop efficient strategies to minimize metal entries into the parts of the plant, and then into the food chain (Ramakrishnan et al. 2021).

Page $13 / 31$ 
XRF results for the remediated soil samples eventuated the existence of the heavy metal (Figures 3 and 4). the peaks showed that though heavy metals were still present in the soil but at low concentrations. Iron was found to have highest peak which shows that vermicopost which consist of microorganism as plant growth promoting bacteria are richer than other treatment. the plant growth can be improved by the presence of this Fe and this can be achieved by increasing the obtainable nutrients or moderating phytohormone levels. The then have the capacity to synthesize phytohormones like gibberellins, auxins and cytokinins which help plant growth and development. (Glick 2010)

Table 6 showed the dispersions of metals across the plant parts (root, seed, leaf and seed). These dispersions of $\mathrm{Cd}$, As and Pb was placed on an interactive relationship with the two locations under the study. The level of significance was determined and it was found that Cd concentration in at the stem of the plants for each location was the highest and is more significant that the concentration in the other part of the plant. For As, the plant part of stem for AM was more significant than the value from other parts of the plant but the concentration at the residual soil showed more significant than the remaining parts. Generally, $\mathrm{Pb}$ showed more increased concentration and the root and stem parts recorded more significant level (Table 6).

Location and each treatment of this study were analysed statically (Table 7), the ranges of the mean difference among the heavy metals were observed and this range differences were used to measure the metal dispersion of the treatment and the plant parts. Considering the permissible level of cadmium, appreciable concentration was obtained $(1.193$ and $1.197 \mathrm{mg} / \mathrm{kg}$ ) by $M$. officinalis $L$ and $S$. acuta respectively with the treatment assisted with vermicompost and PGPB in AK location. These were seen as more significantly different ( $\mathrm{p} \otimes 0.05)$ from the concentration of other treatment in the same location. The obtainable concentration of arsenic was significantly higher ( 3.878 and $4.271 \mathrm{mg} / \mathrm{kg}$ ) with $M$. officinalis $L$ and $S$. acuta respectively with the treatment assisted with CDV+PGPB for both plants in AM location while the lowest value concentration $1.791 \mathrm{mg} / \mathrm{kg}$ was observed in the control treatment of $S$. acuta of AK location. Lead concentration showed more substantial value $11.009 \mathrm{mg} / \mathrm{kg}$ in AM location with M. officinalis L at CDV+PGPB treatment and this followed the same patter with S. acuta in AM location with CDV+PGPB treatment. The ranges obtained with all the metals (Table 6 and 7 ) were used for the canonical discriminant function analysis and these were represented in Figures 2 to 3 . Most phytoremediation plants were reported to bioaccumulate metals in their root parts and to a certain concentration in their leaves or seeds (Kalavrouziotis et al. 2008). This study recorded little differences in the tissue of $S$. acuta. And there were no significant differences in the trend of partitioning of Cadmium, Arsenic and lead in roots and leaves of $S$. acuta. However, the stem generally had more metal concentration. These trends in metal concentration in plant parts could be linked to grasses because the report of Yoon et al. (2006) showed that the concentrations of $\mathrm{Pb}, \mathrm{Cu}$ and $\mathrm{Zn}$ the roots of some grasses (bahia,bermuda and wire grasses) were higher than that of thier leaves contents. $\mathrm{Pd}$ concentration obtained in this study for both both plants were found to be more than the limit value for Pb (10 mg/kg) as mentioned by Sharma et al. (2018), but the contration was lower than Indian standard (250-500 mg/kg) as cited by Alghobar and Suresha (2017) in soil. Cd and As also showed comparable trends. The observable Cd concentrations obtained in this study were also similar with values reported by Sharma et al. (2018) which was $0.79-$ $1.73 \mathrm{mg} / \mathrm{kg}$ and also than the limit value reported by Chang et al. (2014). The main concentration of As in the plants were found to be greater than the recommended value of $0.1 \mathrm{mg} / \mathrm{kg}$ as reported by Shaheen et al. (2016).

The canonical discriminant Analysis (CDA) of the heavy metals ( $\mathrm{Cd}, \mathrm{As}, \mathrm{Pb}$ ) were analyzed and these are presented in Figures 5 and 6 . Ranges of heavy metal interaction with the two locations in respect of all treatment (Table 6) was used to determine the dispersion rate of heavy metal. The range of each metal was used to determine their dispersion and in effect identify the element responsible for the distribution in CDA. Results of a CDA analysis based on root, stem, leaf and seed, element concentrations show a clear distinction between the two locations with different treatment and plant parts.

The ability and capability of plants to extract, accumulate and disperse heavy metals in their tissues are differed from one plant to another (Kacalkova et al. 2015); for this reason, plant selection for heavy metals accumulation depends majorly on their tolerant ability (Rezania et al. 2016). The two plants used for this study had more heavy metal dispersed in their stem (Figure 6) that in their root, leaf and seed (Figure 6). Pb had more distribution of the metals across the plant parts. When plant growth is able to withstand the metal tolerant, the plants will be able to remove more metal from the polluted soil. Few plant species can remove greater contents of metal from polluted soil to their tissue, however, those plant species may produce little biomass and their growth may automatically slow therefore, their capability are not noticeable when selecting plant species for phytoremediation purposes. Selvam and Wong (2008) reported a decline in biomass production of B.napus grown on cadmium polluted soil. Dispersion and accumulation capacity of heavy metals by both plants were scored: $\mathrm{Pb}>\mathrm{As}>\mathrm{Cd}$. This was a pointer that lead was better distributed wide concentrations than that of As and $\mathrm{Cd}$. The polluted soil from AK and Am showed a very multiple variation of dispersions of all metals under investigation, indicating that all metal contents were influenced strongly by the assisted materials (Vermicast and PGPB) used for the study (Manta et al. 2002). Pb distributions were located more in the stem part of the plants, as well as within the root and leaf of the plant. Therefore, the polluted soil from a mining sites are likely the prime contributors to cadmium pollution and this may have effects on its distributions in the native plants used (Xiao et al. 2020).

Generally speaking, in phytoremediation process, phytoextractions requires translocation of metals from the polluted soil to the edible plant parts (Yoon et al. 2006). translocation factor (TF), bioconcentration factor (BCF), biological accumulation coefficient (BAC) and enrichment factor (EF) are all factors responsible for the movement of the heavy metals from the contaminated soil to the plant roots and the capacity of the plant to move the metals from the root to other parts of the plant and vice versa. The capability to accumulate tolerate heavy metals especially from the polluted soil to the roots is useful for phytostabilization and phytoextraction drives (Yoon et al., 2006; Aransiola et al. 2013). When the translocation and bioconcentration factor with biological accumulation coefficient of a Plant is greater than one (TF, BAC and BCF>1), the plant has the ability and are considered to be a phytoextractor. However, if the BCF of a plant is higher than one and the $T F$ is lesser than one (BCF $>1$ and $T F<1)$, the plant is considered to have a phytostabilization (Yoon et al., 2006) $\mathrm{EF}$ is determined as the ratio of plant shoot concentration to the concentration in the soil. It has been established that a hyperaccumulator plant in phytoremediation must have EF> 1 or TF> 1 and also have plant total accumulation higher than $1000 \mathrm{mg} / \mathrm{kg}$.

As shown in Table 8 (remediation of Angwan Kawo soil with M. officinalis $\mathrm{L}$ ) the plant used for this study was suitable for phytoextraction of $\mathrm{Cd}$, As and $\mathrm{Pb}$ because it has $B C F, T F$ and $B A C>1$. Also, $M$. officinalis $L$ shows suitability for phytostabilization of $C d, A s$ and $P b$ with the BCF value ranged from 0.09 to $8.46,0.23$ to 6.0 and 0.22 to 1.16 respectively. TF value ranges for $\mathrm{Cd}$, As and $\mathrm{Pb}$ was 1.59 to $5.68,2.11$ to 4.86 and 0.56 to 3.97 respectively and $\mathrm{BAC}$ has 
valued ranged from 0.27 to 27.69 for $\mathrm{Cd}, 0.50$ to 29.22 for As and 0.18 to 4.51 for $\mathrm{Pb}$. Since the EF for some treatment are higher than 1 but have total accumulation lesser than $1000 \mathrm{mg} / \mathrm{kg}$, M. officinalis $\mathrm{L}$ did not accumulate $\mathrm{Cd}$, As nor Pb above $1000 \mathrm{mg} / \mathrm{kg}$. However, when applying the requirements of reference, if TF and EF are $>1$, the plant can be considered as an accumulator in respect to few of the treatments. According to this criterion $M$. officinalis $L$ is a hyperaccumulator of $\mathrm{Cd}$ and $\mathrm{As}$ ( $\mathrm{TF}=10.76$ and 6.95 respectively) (Table 8).

Table 9 shows accumulation and translocation of $\mathrm{Cd}$, As, and $\mathrm{Pb}$ in $\mathrm{S}$. acuta used for remediation of Angwan Kawo Soil. The BAC value for $S$. acuta are given in table 9. Here, BAC values $>1$ are important because it determines the mechanism of the said plant used for phytoremediation. BAC was greater than 1 for $C d$ and Pb in Soil $(5 \mathrm{~kg})+$ S. acuta + PGPB, Soil $(5 \mathrm{~kg})+$ S. acuta + CDV+ PGPB, Soil (5kg) + S. acuta + GMV+ PGPB, Soil (5kg) + S. acuta + CDV, Soil (5kg) + S. acuta + GMV treatments with 30.86,18.25,19.0,5.20, 13.6 and 1.18, 3.45, 3.92, 1.20 and 2.47 respectively while their BAC was less than 1 only with Soil $(5 \mathrm{~kg})+$ S. acuta treatment (control). Biological accumulation coefficient for As have the values $>1$ in Soil $(5 \mathrm{~kg})+S$. acuta + CDV+ PGPB, Soil $(5 \mathrm{~kg})+S$. acuta $+\mathrm{GMV}+$ PGPB, Soil $(5 \mathrm{~kg})+S$. acuta + CDV, Soil $(5 \mathrm{~kg})+S$. acuta + GMV and this value was lesser than 1 in Soil $(5 \mathrm{~kg})+S$. acuta, Soil $(5 \mathrm{~kg})+S$. acuta + PGPB only. Both $\mathrm{Cd}$, As and $\mathrm{Pb}$ has TF $>1$ for all treatments while EF shows value $>1$ in Soil $(5 \mathrm{~kg})+S$. acuta $+\mathrm{CDV}+\mathrm{PGPB}, \mathrm{Soil}(5 \mathrm{~kg})+S$. acuta $+\mathrm{GMV}+\mathrm{PGPB}, \mathrm{Soil}(5 \mathrm{~kg})+S$. acuta + CDV, Soil $(5 \mathrm{~kg})+S$. acuta + GMV treatments for only Cd and As with 4.75 and 14.90 respectively (Table 9). BCF for Cd, As and Pb ranged from 0.04 to $5.25,0.25$ to 34.14 and 0.24 to 1.44 respectively.

When degradation of contaminant taken up by plant are not rapid, biological accumulation is in play and this result in metal accumulation by plant. In this study as presented in table 9 , none of the plant species showed metal concentrations $>1000 \mathrm{mg} / \mathrm{kg}$ in shoots, i.e., none of them could be considered as a hyperaccumulators. However, the ability of this plant to tolerate and accumulate heavy metals may be useful for phytostabilization. The BCF of Pb in this study for $S$. acuta on Angwan Kawo soil was lower (1.44) than that reported by Kim et al. (2003) in P.redundent (BCF= 58) and more than the value $(B C F=0.004-0.45)$ revealed by Stoltz and Greger (2002). Another report by Shu et al. (2002) reported a bioconcentration factor of 0.1 for lead in $P$. distichum. As presented similar to $\mathrm{Pb}$ accumulation by these plants, they do not accumulate $\mathrm{Cd}$ and $\mathrm{As}$ above $1000 \mathrm{mg} / \mathrm{kg}$ (Table 9). S. acuta growing on the polluted soil of Angwan Kawo was capable of accumulating heavy metals in the roots and shoots, but had BAC, BCF and TF value to be low which is an indicator of plant ability to accumulate heavy metal and translocate them rapidly.

$\mathrm{Cd}$, As and $\mathrm{Pb}$ remediation with $M$. officinalis $\mathrm{L}$ of Angwan Magiro soil were determined and recorded in Table 10. This plant had TF $>1$ in all the treatments considered with $\mathrm{Cd}$ having the highest TF value of 10.5 and the lowest 1.56. This result indicated that the plants translocate metals more effectively from roots part to the shoot (Rungruang et al. 2011). BAC values ranged ( $\mathrm{Cd}=0.54$ to 36.88 , As $=0.34$ to 11.0 and $\mathrm{Pb}=0.36$ to 4.40$)(\mathrm{Table} 10)$. This is a bit different to the range of 1.120 in Fagonia sp. to 1.499 in Gynandris sp. (Rungruang et al. 2011). Only at the plant treated with GMV and PGPB had all the metals with EF greater than one. This shows that EF as a phytoremediation determination index for plants are best suited at this treatment for $M$. officinalis $\mathrm{L}$. Furthermore, only Cd and As had BCF greater than one in just two treatment (Table 10) others were lesser than one. The translocation of Pb to shoot as observed (Table 10) is low and this has been traced to low mobility and of Pb (Yoon et al. 2006, Eid et al. 2020). Table 11 shows the BCF for all the available heavy metals ( $\mathrm{Cd}$, As and $\mathrm{Pb}$ ) but only $\mathrm{Cd}$ had it bioconcentration factor higher than one in all the treatment designed. In fact, $\mathrm{Cd}$ had the highest $\mathrm{BCF}$ (10.37) at Soil $(5 \mathrm{~kg})+$ S. acuta + GMV+ PGPB treatment and the lowest at the plant treated GMV only while As and Pb all had their BCF lower than one in all the treatments designed for Angwan Magiro soil. Sakizadeh et al. (2016) recorded in their studies 0.019 in Pistachio to 0.473 in Spindle tree. These values are lower than values obtained from this study. Singh et al. (2017) in another study recorded BCF of 2.73 in $A$. aspera to 262.13 in $A$. arvensis for Mn. This patter was repeated in EF where only Cd had EF greater than one except in the control with (0.17 EF) and the plant treated with only GMV which had 0.96 as its EF value (Table 11).

Considering the biological accumulation coefficient (BAC) in Table 11, it was discovered that the plant ( $S$. acuta) used for the remediation had the highest $\mathrm{BAC}$ for $\mathrm{Cd}(\mathrm{BAC}=42.5)$ and the lowest was 0.85 . BAC values for As for this plant were greater than 1, except for the control and plant treated with only the PGPB with 0.23 and 0.83 respectively, BAC maximum here was 2.14 (Table 11). The highest BAC for Pb was 2.32 with $S$. acuta while lowest was 0.25 . The translocation factor (TF) which is another index of phytoremediation status for plants ranges ( $\mathrm{Cd}=0.44$ to 4.09 , As $=1.59$ to 4.24 and $\mathrm{Pb} 2.37$ to 3.08 ) ( $\mathrm{Table}$ $4.11)$ in this design. The trend of consideration for EF was that TF was greater than one in all the treatments except only with the control that had TF=0.44 (Table 11) The range of TF is generally higher than the BCF, an indication that most species translocate more Pb and As from roots to shoots (Yoon et al. 2006; Singh et al. 2017). The BCF and TF > 1 also suggests more of the metals in plants than in soil (Nazir et al. 2011; Rungruang et al. 2011) while BCF value $>1$ and TF value $<1$ had been used to evaluate phytostabilization potential of plants (Sudmoon et al. 2015). The current values of TF are not consistent with both previous works. BCF and TF > 1 are adjudged suitable for phytoremediation purposes (Yoon et al. 2006). Based on the values of BCF and TF, S. acuta had $\mathrm{BCF}, \mathrm{TF}$ and $\mathrm{BAC}>1$ in most of the treatment and are such suitable as phytoextractor of $\mathrm{Cd}, \mathrm{As}$ and $\mathrm{Pb}$. Table 4.22 revealed further that $\mathrm{Cd}$ had $\mathrm{BCF}>1$ and $\mathrm{TF}<1$ in the control treatment. This implies that $S$. acuta species are accumulators of $\mathrm{Cd}$ in their roots and are thus suitable for phytostabilization of $\mathrm{Cd}$ (Sudmoon et al. 2015). S. acuta is not considered as hyperaccumulator of Cd, As nor Pb (Table 11). In this study, the two plant species did not show metal concentrations higher than $1000 \mathrm{mg} / \mathrm{kg}$ in the shoots (Tables 8, 9, 10 and 11), i.e. none of them are hyperaccumulators. However, the ability of these plants to tolerate and accumulate heavy metals may be useful for phytostabilization. In general, the three heavy metals were translocated at elevated levels in plant biomass for treatments of CDV and GMV with PGPB.

The achieved $\mathrm{Cd}$, As and $\mathrm{Pb}$ percentage removal with the Angwan Kawo soil remediated with $M$. officinalis $\mathrm{L}$ (Figure 7 ) has the ranged from $34.83-97.07 \%$, $72.5-98.39 \%$ and $78.02-89.55 \%$ respectively. The lowest percentage removal (34.83\%) of the heavy metal Cd was obtained in the soil treated with $M$. officinalis $\mathrm{L}$ alone without any amendments (control) and there was a similar pattern but higher percentage than $\mathrm{Cd}$. As and $\mathrm{Pb}$ both had the lowest percentage removal $(72.5,78.02)$ respectively. Angwan Kawo soil remediated with S. acuta shows similar patter with the one remediated with $M$. Officinalis L. However, highest and best bio-removal percentage $(99.64 \%$ for As) with $S$. acuta was obtained with soil treated with CDV+ PGPB while the unamended soil with S. acuta all recorded lower percentage (Figure 8) for all the metals and Cd had the lowest (73.03\%). Figure 8 shows the percentage removal of Cd, As and $\mathrm{Pb}$ with $M$. officinalis $\mathrm{L}$ on Angwan Magiro soil. The range for $\mathrm{Cd}$, As and $\mathrm{Pb}$ were $70.1-98.35,3.7-90.45$ and $53.36-81.95 \%$ respectively. As had the lowest (3.7\%) of all the value obtained. The highest percentage was recorded for Cd with the soil amended with GMV + PGPB. With S. acuta, there was bio-

Page 15/31 
removal ascending patter with the treatments for all the heavy metals (Figure 10). Generally speaking, both location after the remediation process, had bioremoval of metals to be best with the soil treated with vermicompost together with PGPB. Also, it was observed that both plants did well in the first location (Angwan Kawo) where percentage removal of heavy metal proved to better than the second location (Angwan Magiro) Meanwhile, among the treated soil, the least removal efficiency was realized in the soil amended with PGPB or Vermicompost alone. Likewise, all the treatments were significantly efficient (except for the control) in $\mathrm{Cd}$, As and $\mathrm{Pb}$ removal.

In this study, the potential removal ability observed in the soil assisted with the vermicompost and PGPB may be as a result of the presence of microbes that enhanced the plant growth (Hassan et al. 2020). However, the removal of this metals observed in this work looked similar to that cited by Achal et al. (2011) (94\%) but lower than that obtained with M. officinalis L. on Angwan Magiro soil. This may be attributable to the difference in the experimental conditions, location and or the organisms used for its enhancement. Bio removal of heavy metals when compared are based on the metal pollutants, it was discovered that the order of the removal followed a trend as As $(99.64 \%)>\mathrm{Cd}(98.35 \%)>\mathrm{Pb}(89.55 \%)$ (Figures 7-10). Several mechanisms could have played a role in the removal of the metals from the polluted soil. Looking at the fact that the $\mathrm{pH}$ of the remediated soil was acidic initially and later moved toward neutral, this indicated the likely occurrence of immobilization and solubilization (Turnau, and Kottke 2005).

The severity of remediation was further validated by the structural morphological changes observed using SEM (Figures 11-14). After seven month of remediation process, All the polluted soil before the remediation either by $M$. officinalis $L$. or $S$. acuta exhibited a smooth large compact structural surface which is an indication of metal pollution whereas the remediated soil exhibited small rough structural surfaces validating the remediation of the soil by the two plants. The SEM micrographs of the soil from Angwan Kawo shows more clarity of remediation and showed fine breakage of the soil structure. Various pores/pits and irregularities formed as a result of remediation activity (Figure 11b and 12). These surface changes observed in the SEM micrographs indicated changes the soil structure of the remediated soil with the two plants. This outcome of the structural changes of the soil implied that the plants enhanced by the vermicompost and PGPB were able to have good effects on the remediation of the soil. Although some certain heavy metals are required for growth by plants, extreme concentration of these metals could be toxic to plants. Plants accumulate some nonessential metals in the process where they uptake the necessary ones during their growth and development (Djingova and Kuleff 2000). As this process occurred, the plant changes the structural characteristics of the soil as showed in this study (Figure $14 \mathrm{~g}-\mathrm{h}$ ).

\section{Conclusion}

Phytoremediation is a promising green technology that can be used to remediate heavy metal contaminated soils. In developing countries like Nigeria, this technology can provide low-cost solution to remediate contaminated area, especially abandoned industrial sites (mines and landfills). A PGPB (Bacillus safensis) proved to be effective when combined with vermicompost produced from goat dung and chicken dropping manure. This study has revealed that the native plants ( $M$. officinalis L. and S. acuta) around the polluted mining site of Madaka district of Shikira with Angwan Kawo and Magiro settlements especially when assisted by GMV and CDV, have great potential for phytoextraction and phytostabilization of all metals under study. None of the plants showed potential as hyperaccumulator under current investigation. The results of this study can be used for management and decontamination of Shikira soil polluted with heavy metals using plant species $M$. officinalis L. and S. acuta

\section{Declarations}

\section{Ethical Approval}

Not applicale

\section{Consent to Participate}

Not applicable

\section{Consent to Publish}

All authors have approved the manuscript and agree with its submission to this journal

\section{Authors Contributions}

This work was carried out in collaboration between all authors. Author S.A. Aransiola anchored the field study, gathered the initial data and performed preliminary data analysis designed the study. U.J.J. ljah wrote the protocol and interpreted the data. Author O.P. Abioye and J.D. Bala managed the literature searches and produced the initial draft. All authors read and approved the final manuscript.

\section{Funding}

This research was self-sponsored, NO any external funding.

\section{Competing interest}

Not applicable

Availability of data and materials 
Data available on request from the authors. The data that support the findings of this study are available from the corresponding author upon reasonable request.

\section{References}

Abiya SE, Odiyi BO, Ologundudu FA, Akinnifesi OJ, Akadiri S (2019) Assessment of Heavy Metal Pollution in a Gold Mining Site in Southwestern Nigeria. Biomed J Sci Tech Res 2(2): 22-36

Achal V, Kumari D, Pan X (2011) Bioremediation of chromium contaminated soil by a brown-rot fungus, Gloeophyllum sepiarium. Res. J. Microbiol. 6:1-7.

Acosta JA, Faz A, Martínez S, Zornoza R, Carmona DM (2011) Multivariateget Statistical and GIS-Based Approach to Evaluate Heavy Metals Behavior in Mine Sites for Future Reclamation. Journal of Geochemical Exploration 109(1-3):8-17.

Afonne OJ, Ifediba EC (2020) Heavymetals risks in plant foods - need to step up precautionary measures. Current Opinion Toxicology 22:1-6.

Agbenin JO (1995) Laboratory manual foe soil and plant analysis (selected methods and data analysis). Faculty of Agriculture/ Institute of Agricultural Research, ABU, Zaria, 7-71

Aira M, Monroy F, Domínguez J (2007) Earthworms strongly modify microbial biomass and activity triggering enzymatic activities during vermicomposting independently of the application rates of pig slurry. Science of the total Environment 385(1):252-261.

Alghobar MA, Suresha S (2017) Evaluation of metal accumulation in soil and tomatoes irrigated with sewage water from Mysore city, Karnataka. India. J. Saudi Soc. Agric. Sci 16:49-59. https://doi.org/10.1016/j.jssas.

Ali K, Kiyani AH, Mirza A, Butt CR, Barros TA, Ali R, Iqbal B, Yousaf M (2021) Ornamental plants for the phytoremediation of heavy metals: Present knowledge and future perspectives, Environmental Research, https://doi.org/10.1016/j.envres.2021.110780.

Alirzayeva E, Neumann G, Horst W, Allahverdiyeva Y, Specht A, Alizade V (2017) Multiple mechanisms of heavy metal tolerance are differentially expressed in ecotypes of Artemisia fragrans. Envrion Pollut 220:1024-1035.

Amarlal A, Cruz JV, Cunha RT, Rodrigues A (2006) Baseline levels of metals in volcanic soils of the Azores (Portugal). Journal on Soil \& Sediment Contamination, $15,123-130$

Ameh EG, Omatola OD, Akinde SB (2019) Phytoremediation of toxic metal polluted soil: screening for new indigenous accumulator and translocator plant species, northern Anambra Basin, Nigeria. Environmental Earth Sciences. 78:345 https://doi.org/10.1007/s12665-019-8343-8

APHA (American Public Health Association). (1998). Standard Methods for the Examination of Water and Waste water, American public health association. American, American water works association, water environment federation. Washington, DC.

Aransiola SA, ljah UJJ, Abioye OP (2013) Phytoremediation of Lead Polluted Soil by Glycine max L. Applied and Environmental Soil Science. Article ID 631619, doi: $10.1155 / 2013 / 631619$.

Aransiola SA, ljah UJJ, Abioye OP, Victor-Ekwebelem MO (2021) ANAMMOX in Wastewater Treatment. In: Maddela N.R., García Cruzatty L.C., Chakraborty S. (eds) Advances in the Domain of Environmental Biotechnology. Environmental and Microbial Biotechnology. Springer, Singapore. https://doi.org/10.1007/978981-15-8999-7_15

Atiyeh RM, Lee S, Edwards CA, Arancon NQ, Metzger JD (2002) The influence of humic acids derived from earthworm-processed organic wastes on plant growth. Bioresour Technol 84:7-14

Basta NT, McGowen SL (2004) Evaluation of chemical immobilization treatments for reducing heavy metal transport in a smelter-contaminated soil. Environmental pollution 127:73-82.

Belliturk K, Shrestha P, Görres JH (2015) The Importance of Phytoremediation of Heavy Metal Contaminated Soil Using Vermicompost for Sustainable Agriculture. Journal of Rice Research 3:2. http://dx.doi.org/10.4172/2375-4338.1000e114

Black CA (1965) Method of soil analysis, Agronomy No.9 part 2, Amer. Soc. Agronomy, Madison, Wisconsin Blaylock MJ and Huang JW (2000). Phytoextraction of metals: In phytoremediation of toxic metals. Using plants to clean up the environment. Ed. I Raskin, B.D Ensley, NY Wiley. pp. 53-70.

Bouyoucos GJ (1962) Hydrometer method improved for making particles size analysis of soil. Agronomy Journal, 53:464-465.

Bray RH, Kurtz LT (1945) Determination of total organic and available forms of Phosphorous in soil. Soil Sciences, 59:39-45

Chang CY, Yu HY, Chen JJ, Li FB, Zhang HH, Liu CP (2014) Accumulation of heavy metals in leaf vegetables from agricultural soils and associated potential health risks in the Pearl River Delta, South China. Environ. Monit. Assess 186:1547-1560. https://doi.org/10.1007/s10661-013-3472-0 PMID: 24185814

Daia J, Becquerb T, Rouillec JH, Reversata G, Reversata FB (2004) Heavy Metal Accumulation by Two Earthworm Species and Its Relationship to Total and DTPA-Extractable Metals in Soils. Soil Biology \& Biochemistry 36:91-98.

Page $17 / 31$ 
Dinev N, Banov M, Nikova I (2008) Monitoring and Risk Assessment of Contaminated Soils. General and Applied Plant Physiology 34:389-396.

Djingova R, Kuleff I (2000) Instrumental techniques for trace analysis, in Trace Elements: Their Distribution and Effects in the Environment, Journal Pharmaceutical and Vernet, Ed., Elsevier, London, UK.

Donkor AK, Bonzongo JCJ, Nartey VK, Adotey DK (2005) Heavy metals in sediments of the gold mining impacted Pra River basin, Ghana, West Africa. Soil and Sediment Contamination 14(6):479-503.

Dzombak DA, Morel FMM (1987) Adsorption of Inorganic Pollutants in Aquatic Systems. Journal of Hydraulic Engineering 113:430-475. http://dx.doi.org/10.1061/(ASCE)0733-9429(1987) 113:4(430)

Eckert D, Sims JT (1995) Recommended soil pH and lime requirement tests. http://ag.udel.edu/extension/information/prod_agric/chap3-95.htm

Eid, EM, Galal TM, Sewelam NA, Talha NI, Abdalla SM (2020). Phytoremediation of heavy metals by four aquatic macrophytes and their potential use as contamination indicators: a comparative assessment. Environmental Science and Pollution Research (2020) 27:12138-12151

https://doi.org/10.1007/s11356-020-07839-9

Elekes CC (2014) Eco-technological solutions for the remediation of polluted soil and heavy metal recovery. In: Hernández-Soriano MC (ed) Environmental risk assessment of soil contamination. In Tech, Rijeka 309-335

Elvira C, Goicoechea M, Sampedro L, Mato S, Nogales R (1996) Bioconversion of solid paper-pulp mill sludge by earthworms. Bioresource Technology 57(2):173-177.

Emenike CU, Agamuthu P, Fauziah SH (2017) Sustainable remediation of heavy metal polluted soil: a biotechnical interaction with selected bacteria species. Journal of Geochemical Exploration, 182:275-278

Federal Ministry of Health, FMH, Nigeria (FMH, 2015). Lead poison led to death of 28 children in the year 2015

Fernández-Gómez MJ, Nogales R, Insam H, Romero E, Goberna M (2010) Continuous-feeding vermicomposting as a recycling management method to revalue tomato-fruit wastes from greenhouse crops. Waste management 30(12):2461-2468.

Fijalkowski K, Kacprzak M, Grobelak A, Placek A (2012) The Influence of Selected Soil Parameters on the Mobility of Heavy Metals in Soils. Inzynieria Ochrona Srodowiska 5:81-92.

Galadima A, Garba ZN (2011) Recent Issues in Environmental Science. "Including incidences and reports from Nigeria, Lap Lambert Academic Publishers.

Garg V, Gupta R (2011) Optimization of cow dung spiked pre-consumer processing vegetable waste for vermicomposting using Eisenia fetida, Ecotoxicology and Environmental Safety 74(1):19-24.

Glick BR (2010) Using soil bacteria to facilitate phytoremediation. Biotechnology Advances 28(3):367-374

Hait S, Tare V (2011) Vermistabilization of primary sewage sludge. Bioresource Technology 102(3):2812-2820.

Hassan A, Agamuthu P, Ahmed A, Innocent O, Fauziah SH (2020) Effective bioremediation of heavy metal-contaminated landfill soil through bioaugmentation using consortia of fungi J. Soils Sedim. 1-15, https://doi.org/10.1007/ s11368-019-02394-4.

Herwijnen RV, Hutchings TR, Al-Tabbaa A, Moffat AJ, Johns ML (2007) Remediation of metal contaminated soil with mineral-amended composts. Environmental Pollution 347-354.

Ikhumetse AA, Abioye OP Aransiola SA (2019) Biosorption Potential of Bacteria on Lead and Chromium in Groundwater Obtained from Mining Community. Acta Scientific Microbiology 2(6):123-137.

Jadia CD, Fulekar MH (2009) Phytoremediation of heavy metals: Recent Techniques. African Journal of Biotechnol, 8, 921-928

Kacalkova L, Tlustoa P, Szakova J (2015) Phytoextraction of risk elements by willow and poplar trees. International Journal of Phytoremediation 17:414-421.

Kai W, Jie Z, Zhiqiang Z, Huagang H, Tingqiang L, Zhenli H, Xiaoe Y, Ashok A (2012) Chicken manure vermicompost (PMVC) can improve phytoremediation of Cd and PAHs co-contaminated soil by Sedum alfredii. J Soils Sediments 12:1089-109

Kalavrouziotis IK, Robolas P, Koukoulakis PH, Papadopoulos AH (2008) Effects of municipal wastewater on the macro- and microelements status of soil and of Brassica oleracea var. Italica, and B. oleracea var Gemmifera, Agricultura and Water Management 95:419-426.

Kaplan M (2016) The national master plan for agricultural development in Suriname. Final report. Kaplan Planners Ltd. Regional and Environmental Planning. https://www. share4dev.info/kb/documents/5426.pdf

Kaur A, Singh J, Vig AP, Dhaliwal SS Rup PJ (2010) Cocomposting with and without Eisenia fetida for conversion of toxic paper mill sludge to a soil conditioner. Bioresource Technology 101(21):8192-8198. 
Khwairakpam M, Bhargava R (2009) Vermitechnology for sewage sludge recycling. Journal of Hazardous Materials 161:948-954.

Kim IS, Kang KH, Johsen-Green P, Lee EJ (2003) Investigation of heavy metal accumulation in Polygonum thunbergii for phytoextraction. Environmental Pollution, 126:235-243.

Koretsky C (2000) The significance of surface complexation reactions in hydrologic systems: a geochemist's perspective. Journal of Hydrology 230(34):127-171. doi:10. 1016/S0022-1694(00)00215-8.

Lorestani B, Cheraghi M, Yousefi N (2011) Phytoremediation potential of native plants growing on a heavy metals contaminated soil of coppermine in Iran. World Academic Science and Engineering Technology 5:341-346

Maddela NR, Kakarla D, García LC, Chakraborty S, Venkateswarlu K, Megharaj M (2020) Cocoa-laden cadmium threatens human health and cacao economy: a critical view. Sci. Total Environ 720:137645.

Malafaia G, da Costa Estrela D, Guimarães AT, de Araújo FG, Leandro WM, de Lima Rodrigues AS (2015) Vermicomposting of different types of tanning sludge (liming and primary) mixed with cattle dung, Ecological Engineering, 85:301-306.

Manta DS, Angelone M, Bellanca A, Neri R, Sprovieri M (2002) Heavy metals in urban soils: a case study from the city of Palermo (Sicily), Italy, Science of the Total Environment 300(1-3):229-243

Martinez T, Lartigue J, Avilaperez P, Navarrete M, Zarazua G, Lopez C, Cabrera L, Nadal M, Schuhmacher M, Domingo JL, (2004) Metal pollution of soils and vegetation in a petrochemical industry. Science of Total Environment, 321:59-69.

Mousavi SA, Sader SR, Farhadi F, Faraji M, Falahi F (2019) Vermicomposting of grass and newspaper waste mixed with cow dung using Eisenia fetida: physicochemical changes, Global NEST Journal 22(1):8-14, https://doi.org/10.30955/gnj.003151

Nazir A, Malik RN, Ajaib M, Khan N, Siddiqui MF (2011) Hyperaccumulators of heavy metals of industrial areas of Islamabad and Rawalpindi. Pakistan Journal of Botany 43(4):1923-1933

Ndegwa PM, Thompson SA Das KC (2000) Effects of stocking density and feeding rate on vermicomposting of biosolids. Bioresource Technology 71(1):5-12.

Nejad ZD, Jung MC, Kim KH (2017) Remediation of soils contaminated with heavy metals with an emphasis on immobilization technology. Environ Geochem Health, DOI 10.1007/s10653-017-9964-z

Nordberg G, Nogawa K, Nordberg M, Friberg L (2007) Cadmium. In: Handbook on toxicology of metals. Nordberg G, Fowler B, Nordberg M, Friberg, L editors New York: Academic Press, 65-78.

Plaza C, Nogales R, Senesi N, Benitez E, Polo A (2008) Organic matter humification by vermicomposting of cattle manure alone and mixed with two-phase olive pomace. Bioresource Technology 99(11):5085-5089.

Ramakrishnan B, Maddela NR, Venkateswarlu K, Megharaj M (2021) Organic farming: Does it contribute to contaminant-free produce and ensure food safety? Science of the Total Environment 769:145079

Rezania S, Taib SM, Md Din MF, Dahalan FA, Kamyab H (2016) Comprehensive review on phytotechnology: heavy metals removal by diverse aquatic plants species from wastewater. Journal of Hazardous Material 318:587-599.

Rungruang N, Babel S, Parkpian P (2011) Screening of potential hyperaccumulator for cadmium from contaminated soil. Desalin Water Treat 32:19-26

Selvam A, Wong JW (2008) Phytochelatin synthesis and cadmium uptake of Brassica napus. Environmental Technology 29:765-773.

Shaheen N, Irfan NM, Khan IN, Islam S, Islam MS, Ahmed MK (2016) Presence of heavy metals in fruits and vegetables: Health risk implications in Bangladesh Chemosphere. 152:431-438. https://doi.org/ 10.1016/J.CHEMOSPHERE.2016.02.060 PMID: 27003365

Sharma S (2003) Municipal solid waste management through vermicomposting employing exotic and local species of earthworms. Bioresource Technology 90(2):169-173.

Sharma S, Nagpal AK, Kaur I (2018) Heavy metal contamination in soil, food crops and associated health risks for residents of Ropar wetland, Punjab. India and its environs. Food Chem. 255:15-22. https://doi.org/10.1016/j.foodchem.2018.02.037 PMID: 29571461

Shu WS, Xia HP, Zhang ZQ (2002) Use of Vetiver and Three Other Grasses for Revegetation of Pb/Zn Mine Tailings: Field Experiment. International Journal of Phytoremediation 4:47-57. http://dx.doi.org/10.1080/15226510208500072

Singh J, Kalamdhad AS (2013) Chemical Speciation of Heavy Metals in Compost and Compost Amended Soil, A Review. International Journal of Environmental Engineering Research 2:27-37.

Singh N, Kaur M, Katnoria JK (2017) Analysis on bioaccumulation of metals in aquatic environment of Beas River Basin: a case study from Kanli wetland. GeoHealth 1:93-105 
Soobhany N, Mohee R, Garg VK (2015) Recovery of nutrient from municipal solid waste by composting and vermicomposting using earthworm Eudrilus eugeniae, Journal of Environmental Chemical Engineering 3(4), 2931-2942.

Stoltz E, Greger M (2002) Accumulation properties of As, Cd, Cu, Pb and Zn by four wetland plants species growing on submerged mine tailings. Environmental Experimental Botany 47:271-80

Sudmoon R, Neeratanaphan L, Thamsenanupap P, Tanee T (2015) Hyperaccumulation of cadmium and DNA changes in popular vegetable, Brassica chinensis L. Int J Environ Res 9(2):433-438

Sunitha R, Mahimairaja S, Bharani A, Gayathri P (2014) Enhanced Phytoremediation Technology for Chromium Contaminated Soils using Biological Amendments. International Journal of Science and Technology 3:153-162.

Takac P, Szabova T, Kozakova L, Benkova M (2009) Heavy Metals and Their Bioavailability from Soils in the Long-Term Polluted Central Spis Region of SR. Plant, Soil and Environment 55:167-172

Tognetti C, Laos F, Mazzarino MJ, Hernandez MT (2005) Composting vs. vermicomposting: a comparison of end product quality, Compost Science \& Utilization, 13(1):6-13.

Turnau K, Kottke I (2005) Fungal activity as determined by microscale methods with special emphasis on interactions with heavy metals. Mycol. Series 23:287.

Ullah A, Mushtaq H, Ali H, Farooq M, Munis H, Tariq JM, Chaudhary HJ (2015) Diazotrophs-assisted phytoremediation of heavy metals: a novel approach. Environ Sci Pollut Res 22:2505-2514 DOI 10.1007/s11356-014-3699-5

USEPA (1996) Air Quality Criteria for Particulate Matter. VI. United States of Environmental Protection Agency. Research Triangle, NC Environmental Criteria and Assessment Office. EPA Report No: EPA/600/P-95/001.

Vig AP, Singh J, Wani SH, Dhaliwal SS (2011) Vermicomposting of tannery sludge mixed with cattle dung into valuable manure using earthworm Eisenia fetida (Savigny). Bioresource Technology 102(17):7941-7945.

Walkley A, Black IA (1934) An examination of the Degtjareff method for determining organic carbon in soils: Effect of variations in digestion conditions and of inorganic soil constituents. Soil Sciences, 63:51-263.

Wang K, Zhang J, Zhu Z, Huang H, Li T (2012) Pig manure vermicompost (PMVC) can improve phytoremediation of Cd and PAHs co-contaminated soil by Sedum alfredii. Journal of Soils and Sediments 12:1089-1099.

Wani K, Rao R (2013) Bioconversion of garden waste, kitchen waste and cow dung into value-added products using earthworm Eisenia fetida, Saudi Journal of Biological Sciences 20(2):149-154.

Xiao X, Zhang J, Wang H (2020) Distribution and health risk assessment of potentially toxic elements in soils around coal industrial areas: a global metaanalysis. Science of the Total Environment 713.

Yadav A, Garg V (2011) Recycling of organic wastes by employing Eisenia fetida, Bioresource Technology 102(3): 2874-2880.

Yobouet YA, Adouby K, Trokourey A, Yao B (2010) Cadmium, Copper, Lead and Zinc Speciation in Contaminated Soils. International Journal of Engineering Science and Technology 2:802-812.

Yoon J, Ceo X, Zhou Q, Ma LQ (2006) Accumulation of Pb, Cu, and Zn in native plants growing on a contaminated Florida site. Science Total Environmental $368: 456-464$

\section{Figures}




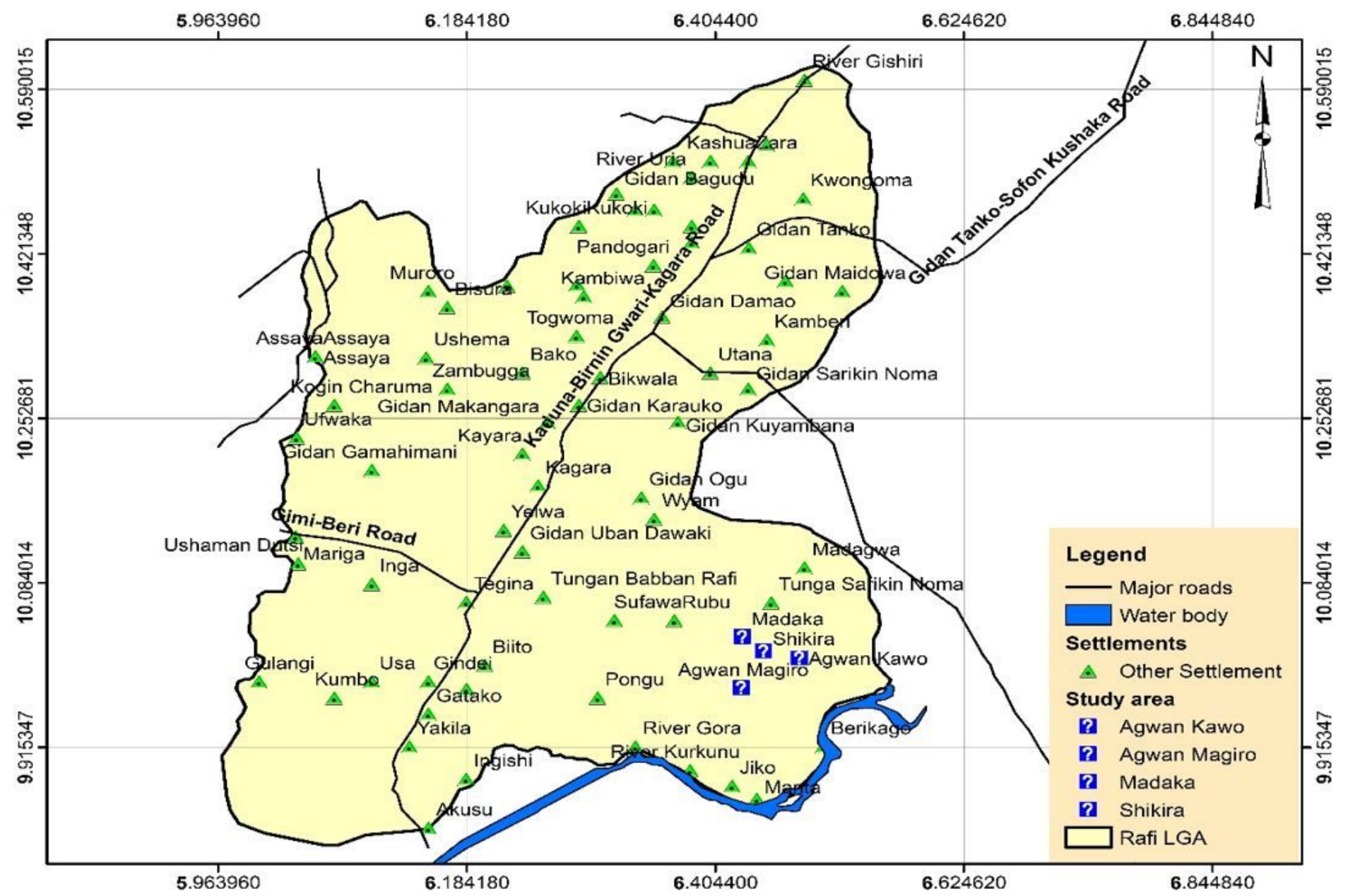

Figure 1

The Study Area (Madaka district, Shikira Community) Rafi LGA, Niger State, Nigeria. Note: The designations employed and the presentation of the material on this map do not imply the expression of any opinion whatsoever on the part of Research Square concerning the legal status of any country, territory, city or area or of its authorities, or concerning the delimitation of its frontiers or boundaries. This map has been provided by the authors. 

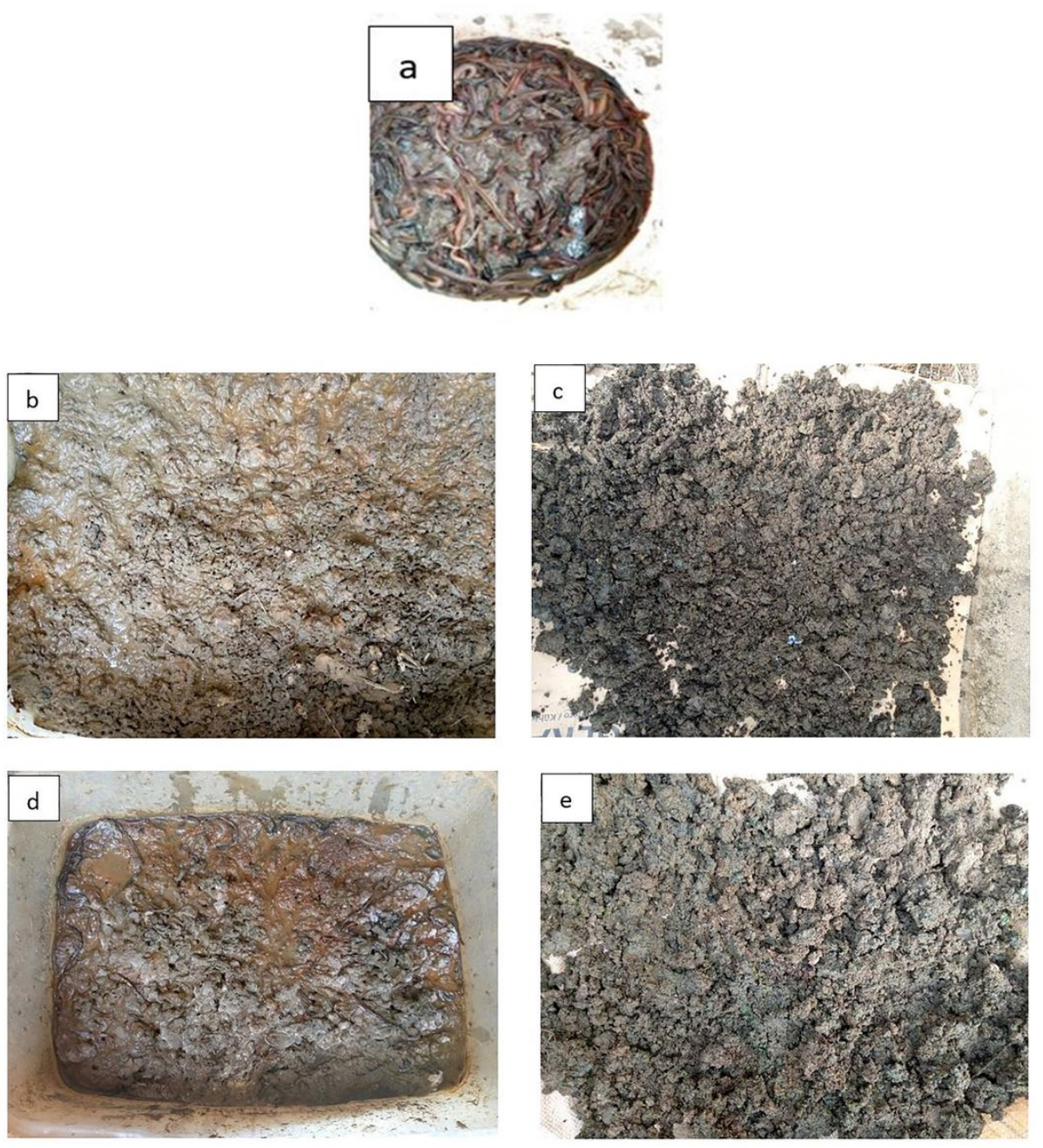

\section{Figure 2}

a. Earthworms (Eisenia foetida) Used for the Study. b. Production Stage of Goat Manure Vermicast, c. Produced Goat Manure Vermicast, d. Production Stage of Chicken Dropping Vermicast, e. Produced Chicken Dropping Vermicast 


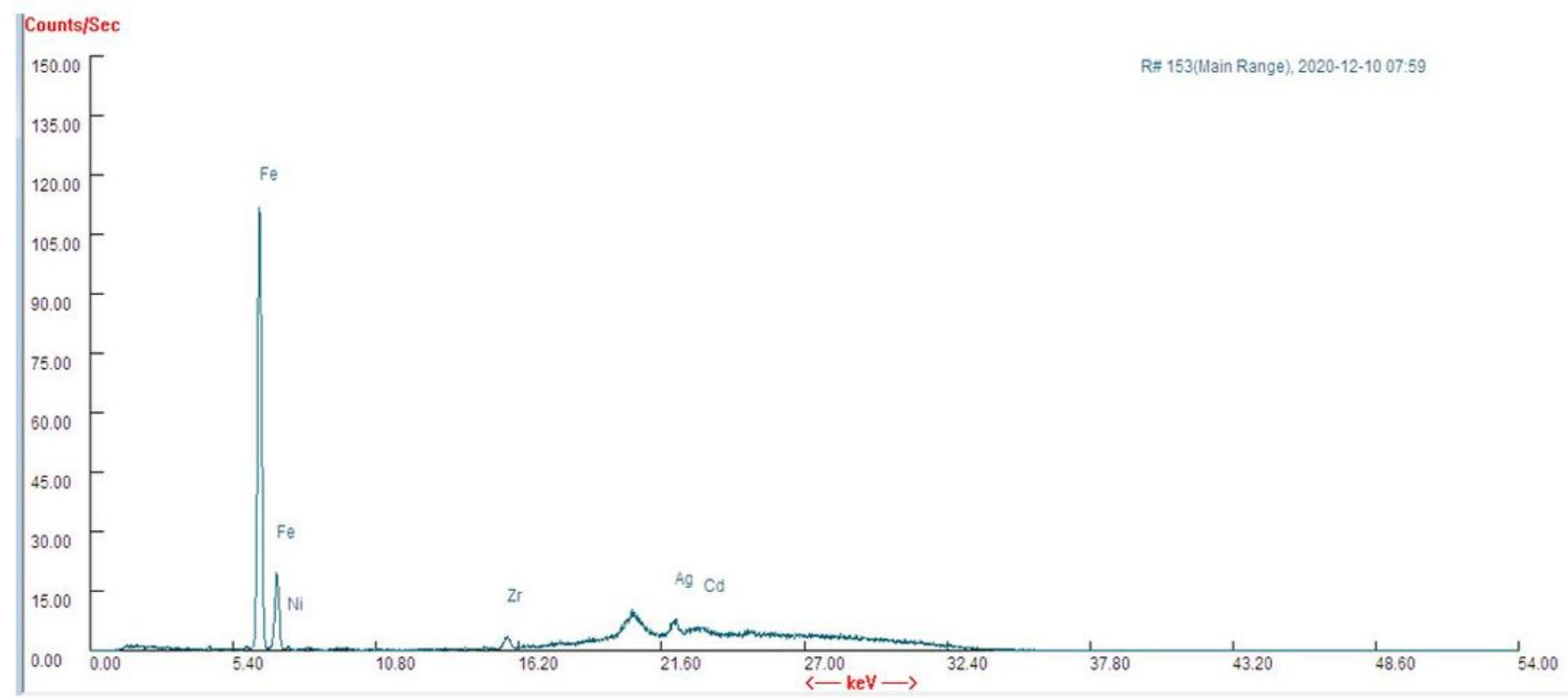

\begin{tabular}{|c|c|c|c|c|c|c|c|c|c|}
\hline $\begin{array}{l}\text { Reading } \\
\text { No }\end{array}$ & Type & Duration & Units & $\mathrm{Fe}$ & $\begin{array}{l}\mathrm{Fe} \\
\text { Error }\end{array}$ & $\mathrm{Ag}$ & $\begin{array}{l}\mathrm{Ag} \\
\text { Error }\end{array}$ & $\mathrm{Rh}$ & $\begin{array}{l}\text { Rh } \\
\text { Error }\end{array}$ \\
\hline 153 & $\begin{array}{l}\text { Precious } \\
\text { Metals }\end{array}$ & 30.63 & $\%$ & 95.214 & 0.792 & 0.38 & 0.1 & 0.471 & 0.073 \\
\hline $\mathrm{Ru}$ & Ru Error & $\mathrm{Co}$ & $\begin{array}{l}\text { Co } \\
\text { Error }\end{array}$ & $\mathrm{Mn}$ & $\begin{array}{l}\mathrm{Mn} \\
\text { Error }\end{array}$ & $\mathrm{Ti}$ & $\begin{array}{l}\mathrm{Ti} \\
\text { Error }\end{array}$ & $\mathrm{Pb}$ & $\begin{array}{l}\mathrm{Pb} \\
\text { Error }\end{array}$ \\
\hline 0.123 & 0.035 & 1.102 & 0.304 & 0.779 & 0.157 & 1.551 & 0.266 & 0.127 & 0.063 \\
\hline
\end{tabular}

Figure 3

XRF Spectrum of Angwan Kawo Remediation Soil 


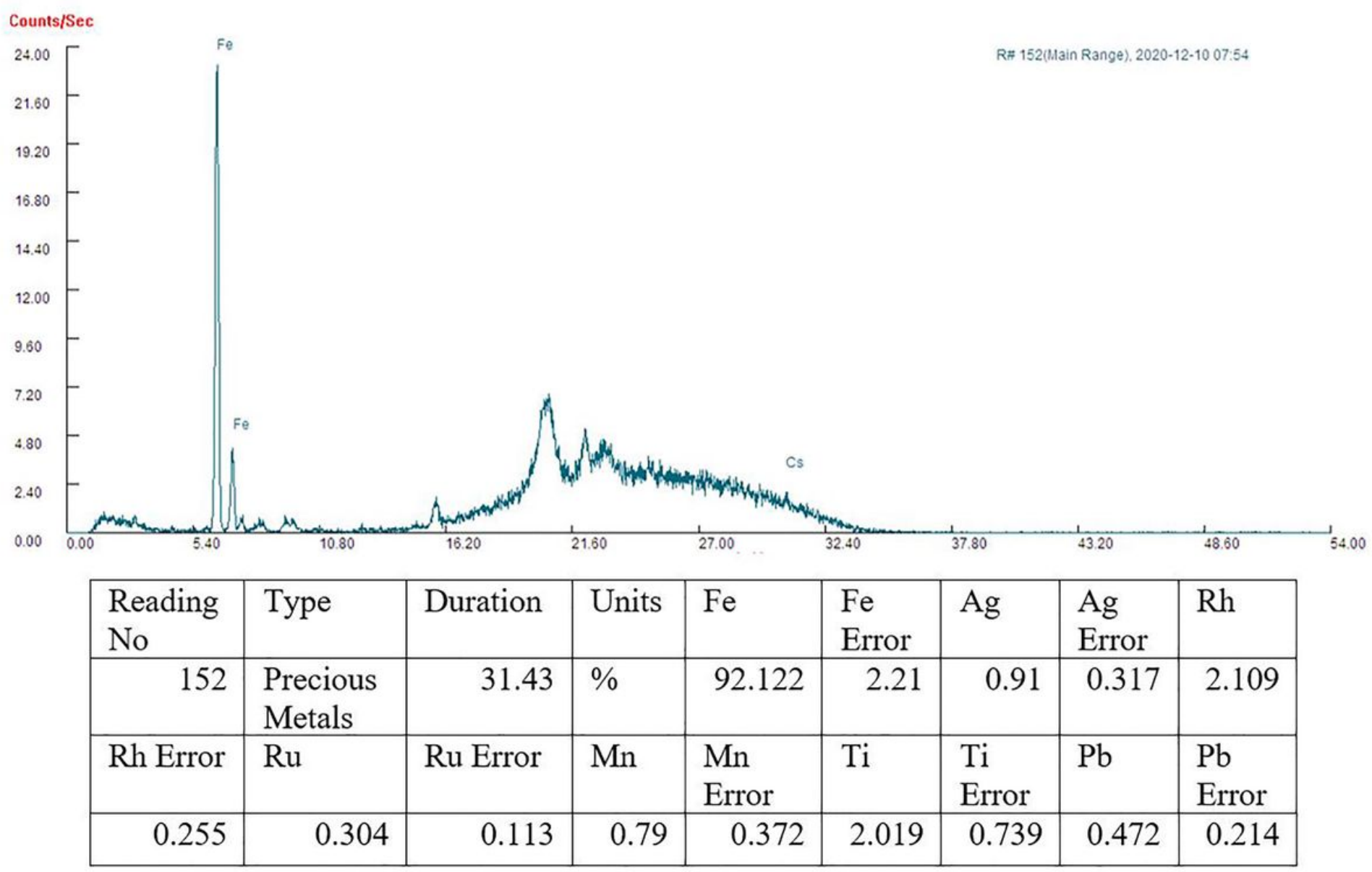

Figure 4

XRF Spectrum of Angwan Magiro Remediation Soil 

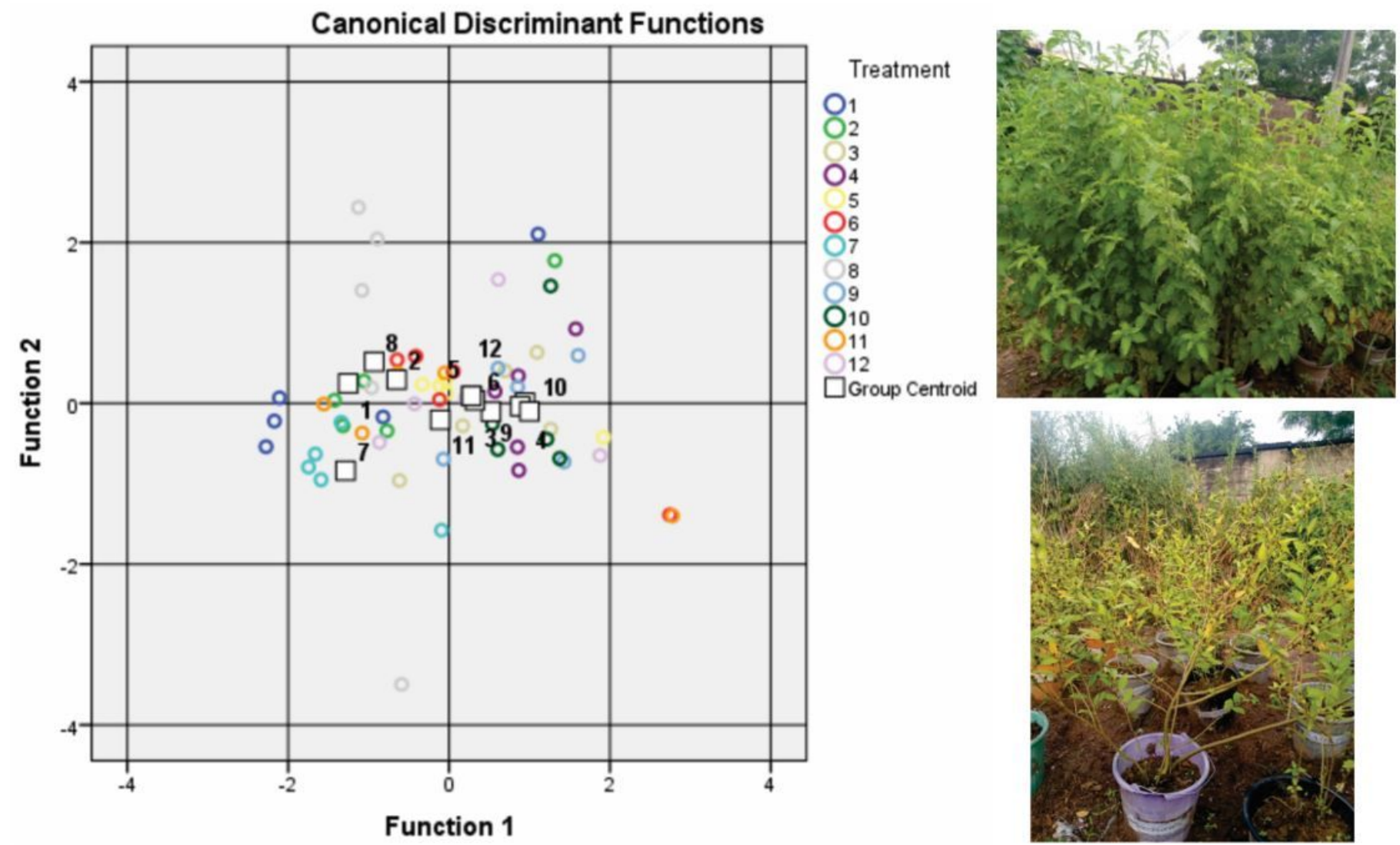

Figure 5

Canonical Discriminant Analysis (CDA) of Heavy metal for All treatment of AK and AM Polluted Soil Key: $1=$ Soil $(5 \mathrm{~kg})+M$. officinalis $L, 2=$ Soil $(5 \mathrm{~kg})+\mathrm{M}$. officinalis $L+P G P B, 3=$ Soil $(5 \mathrm{~kg})+M$. officinalis $L+C D V+P G P B, 4=$ Soil $(5 \mathrm{~kg})+M$. officinalis $L+G M V+P G P B, 5=S o i l(5 k g)+M$. officinalis $L+C D V, 6=$ Soil $(5 \mathrm{~kg})+$ M. officinalis $L+\mathrm{GMV}, 7=$ Soil $(5 \mathrm{~kg})+\mathrm{S}$. acuta, $8=$ Soil $(5 \mathrm{~kg})+\mathrm{S}$. acuta + PGPB, $9=$ Soil $(5 \mathrm{~kg})+\mathrm{S}$. acuta + CDV+ PGPB, $10=$ Soil $(5 \mathrm{~kg})+\mathrm{S}$. acuta + GMV+ PGPB, $11=$ Soil $(5 \mathrm{~kg})+$ S. acuta + CDV, $12=$ Soil $(5 \mathrm{~kg})+$ S. acuta + GMV. 

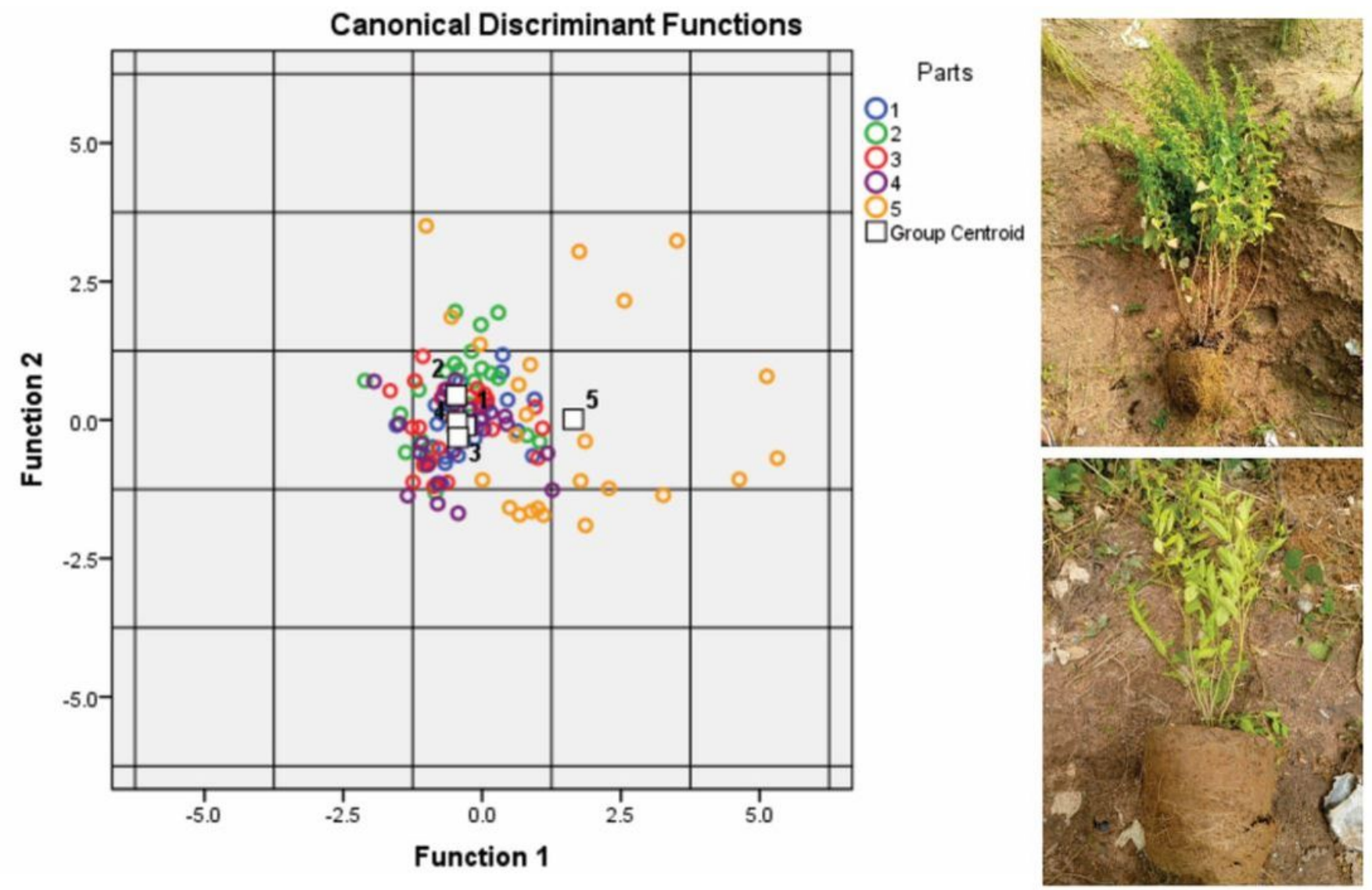

Figure 6

Canonical Discriminant Analysis (CDA) of Plant Parts for Cd, As and Pb in AK and AM Polluted Soil. Key: Key: $1=$ Root, $2=$ Stem, $3=$ Leaf, $4=$ Seed, $5=$ Soil.

$\square \mathrm{Cd} \square \mathrm{As} \square \mathrm{Pb}$

120

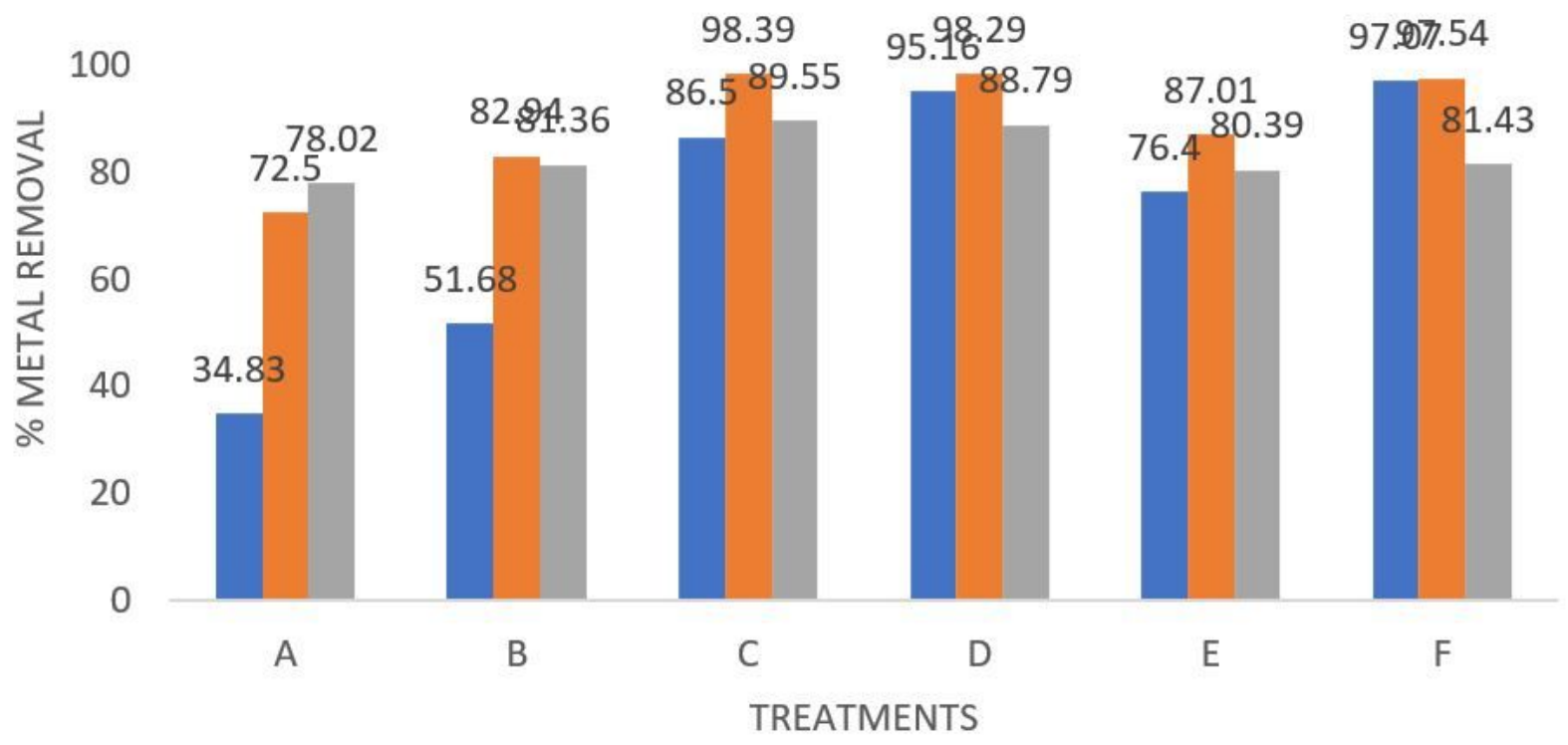

Figure 7

Percentage of Heavy Metal Bio-removal Efficiency with M. officinalis $L$ of Angwan Kawo Soil $A=S o i l(5 k g)+M$. officinalis $L, B=S o i l(5 k g)+M$. officinalis $L+$ $P G P B, C=$ Soil $(5 \mathrm{~kg})+M$. officinalis $L+C D V+P G P B, D=$ Soil $(5 k g)+M$. officinalis $L+G M V+P G P B, E=S o i l(5 k g)+M$. officinalis $L+C D V, F=S o i l(5 k g)+M$. 
$\square \mathrm{Cd}=\mathrm{As} \mathrm{Pb}$

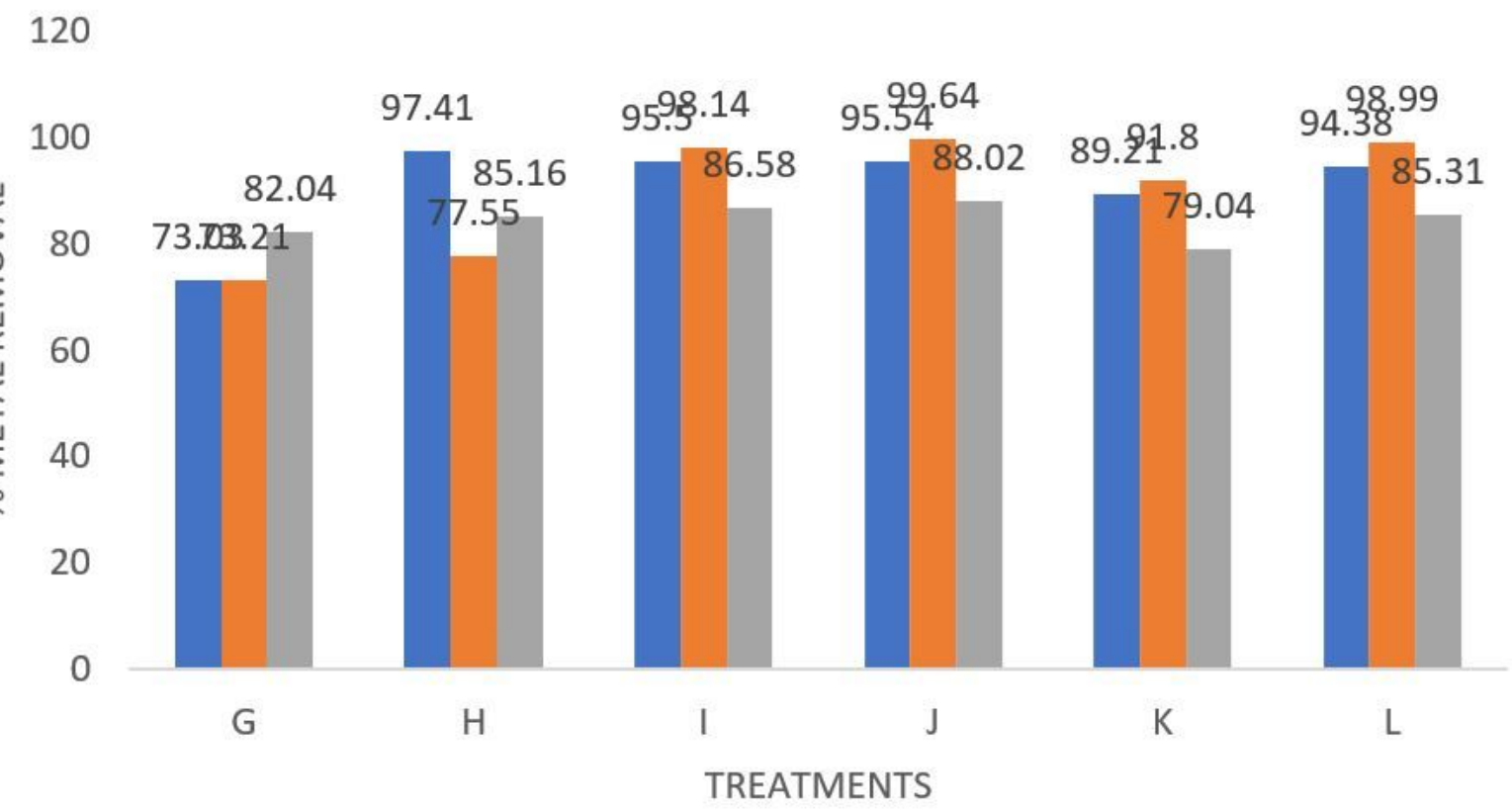

\section{Figure 8}

Percentage of Heavy Metal Bio-removal Efficiency with S. acuta of Angwan Kawo Soil G= Soil $(5 \mathrm{~kg})+\mathrm{S}$. acuta, H= Soil $(5 \mathrm{~kg})+\mathrm{S}$. acuta + PGPB, I= Soil $(5 \mathrm{~kg})+$ S. acuta + CDV+ PGPB, J= Soil (5kg) + S. acuta + GMV+ PGPB, K= Soil (5kg) + S. acuta + CDV, L= Soil (5kg) + S. acuta + GMV

\section{$\square \mathrm{Cd}$ As $\mathrm{Bb}$}

120

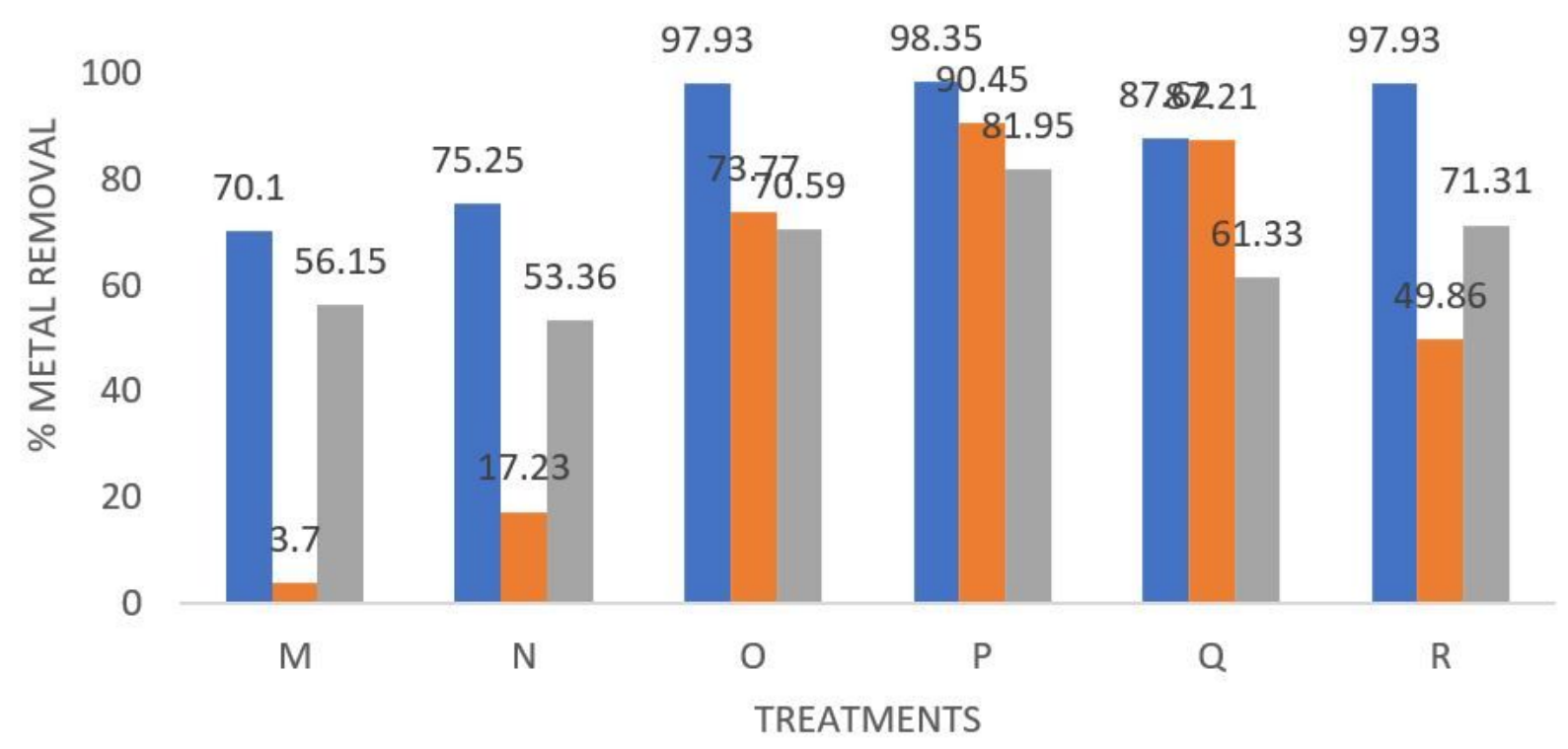

Figure 9

Percentage of Heavy Metal Bio-removal Efficiency with M. officinalis $L$ of Angwan Magiro Soil M= Soil $(5 \mathrm{~kg})+M$. officinalis $L, N=S o i l(5 \mathrm{~kg})+M$. officinalis $L+$ PGPB, $\mathrm{O}=$ Soil $(5 \mathrm{~kg})+\mathrm{M}$. officinalis $L+C D V+P G P B, P=$ Soil $(5 \mathrm{~kg})+$ M. officinalis $L+G M V+P G P B, Q=$ Soil $(5 \mathrm{~kg})+M$. officinalis $L+C D V, R=$ Soil $(5 \mathrm{~kg})+M$. officinalis $L+G M V$ 
120

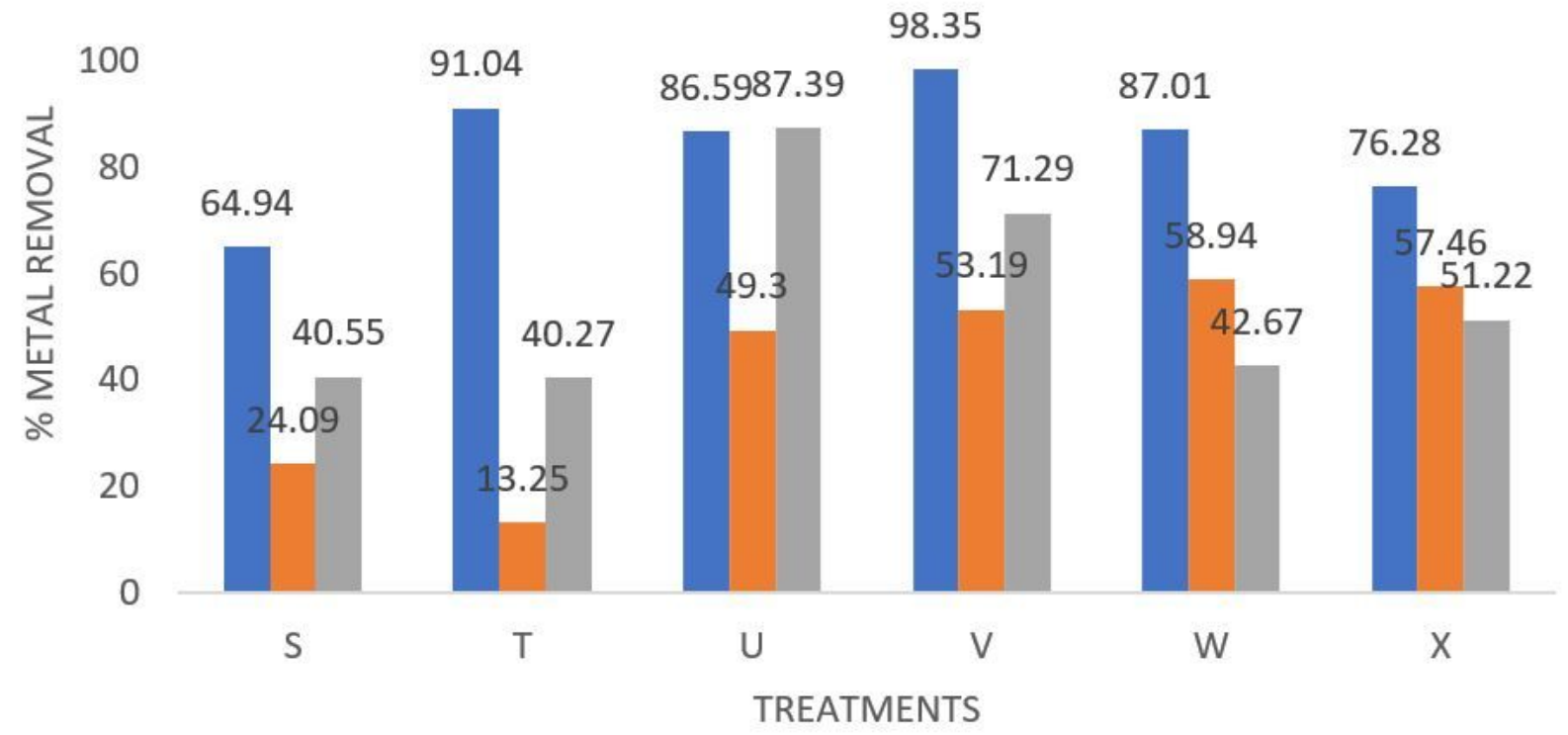

Figure 10

Percentage of Heavy Metal Bio-removal Efficiency with S. acuta of Angwan Magiro Soil S= Soil $(5 \mathrm{~kg})+\mathrm{S}$. acuta, $\mathrm{T}=\mathrm{Soil}(5 \mathrm{~kg})+\mathrm{S}$. acuta + PGPB, U= Soil $(5 \mathrm{~kg})$ + S. acuta + CDV+ PGPB, V= Soil $(5 \mathrm{~kg})+\mathrm{S}$. acuta + GMV+ PGPB, W= Soil $(5 \mathrm{~kg})+\mathrm{S}$. acuta + CDV, X= Soil (5kg) + S. acuta + GMV
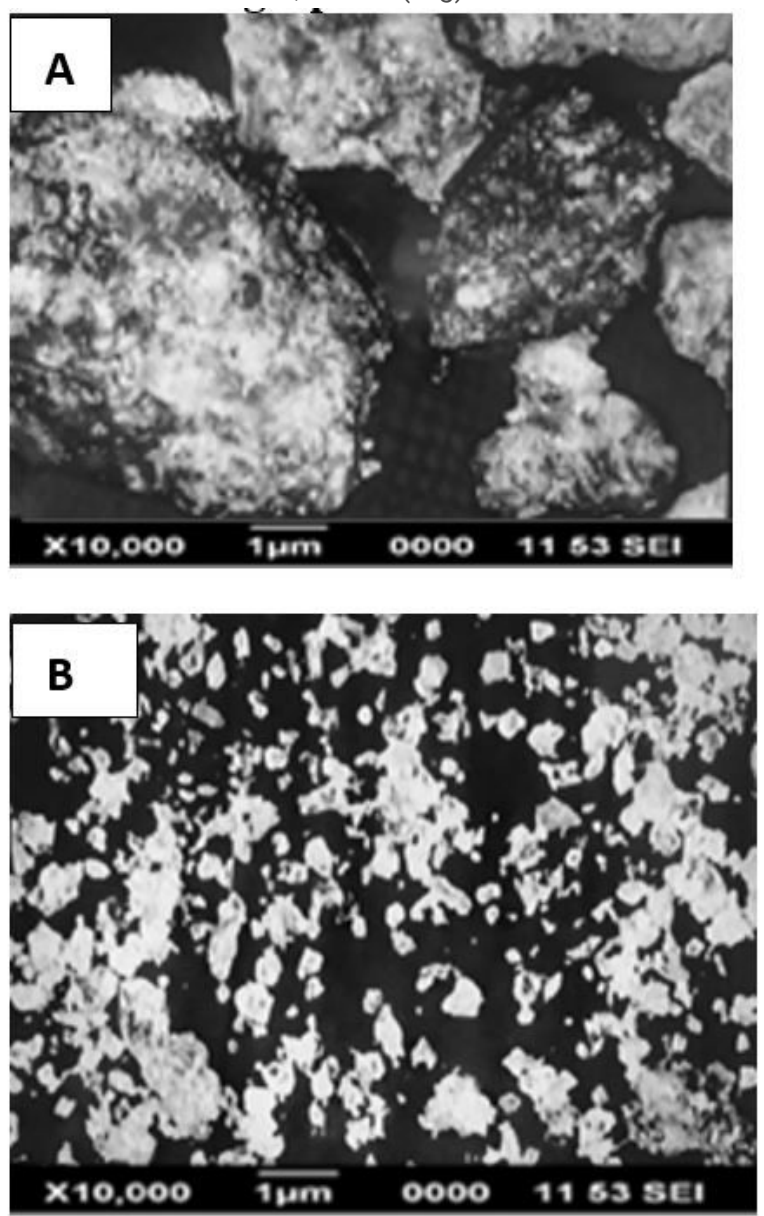
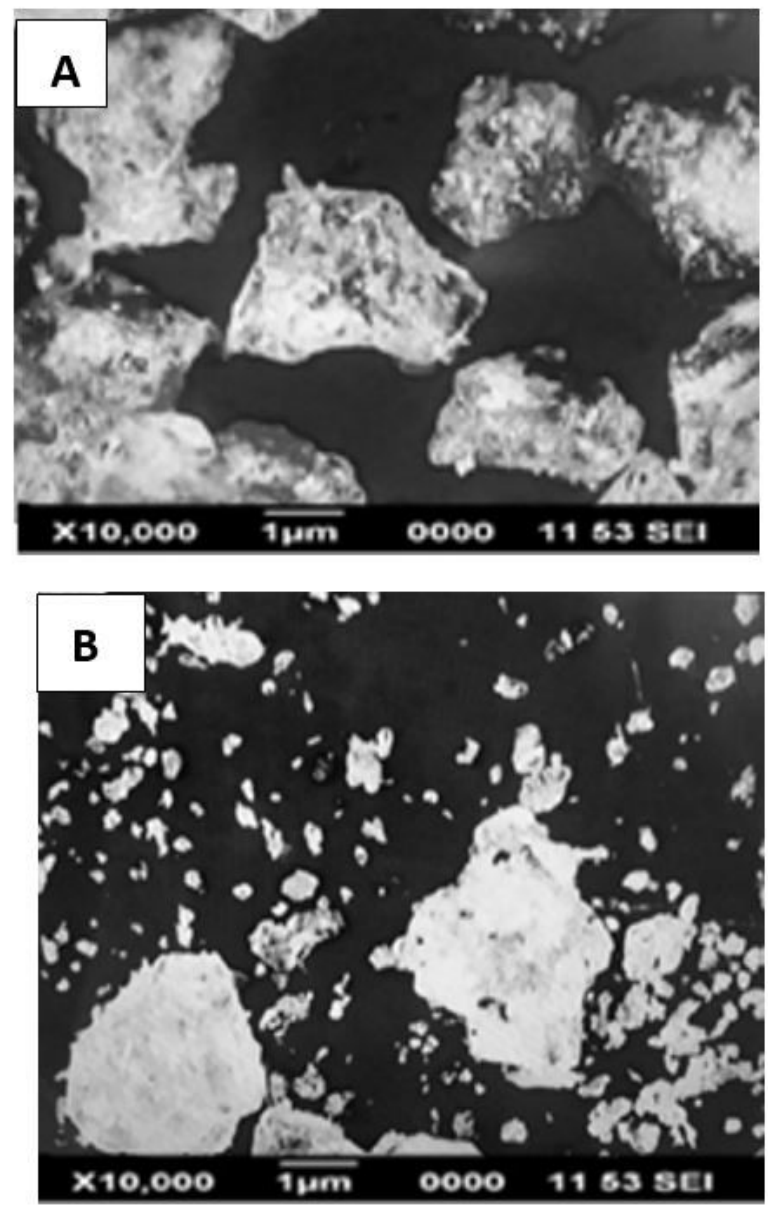
a. SEM Micrographs of the Polluted Soil of Angwan Kawo Before Remediation with M. officinalis L. b. SEM Morphological Appearance of the Remediated Soil of Angwan Kawo with M. officinalis L After Remediation
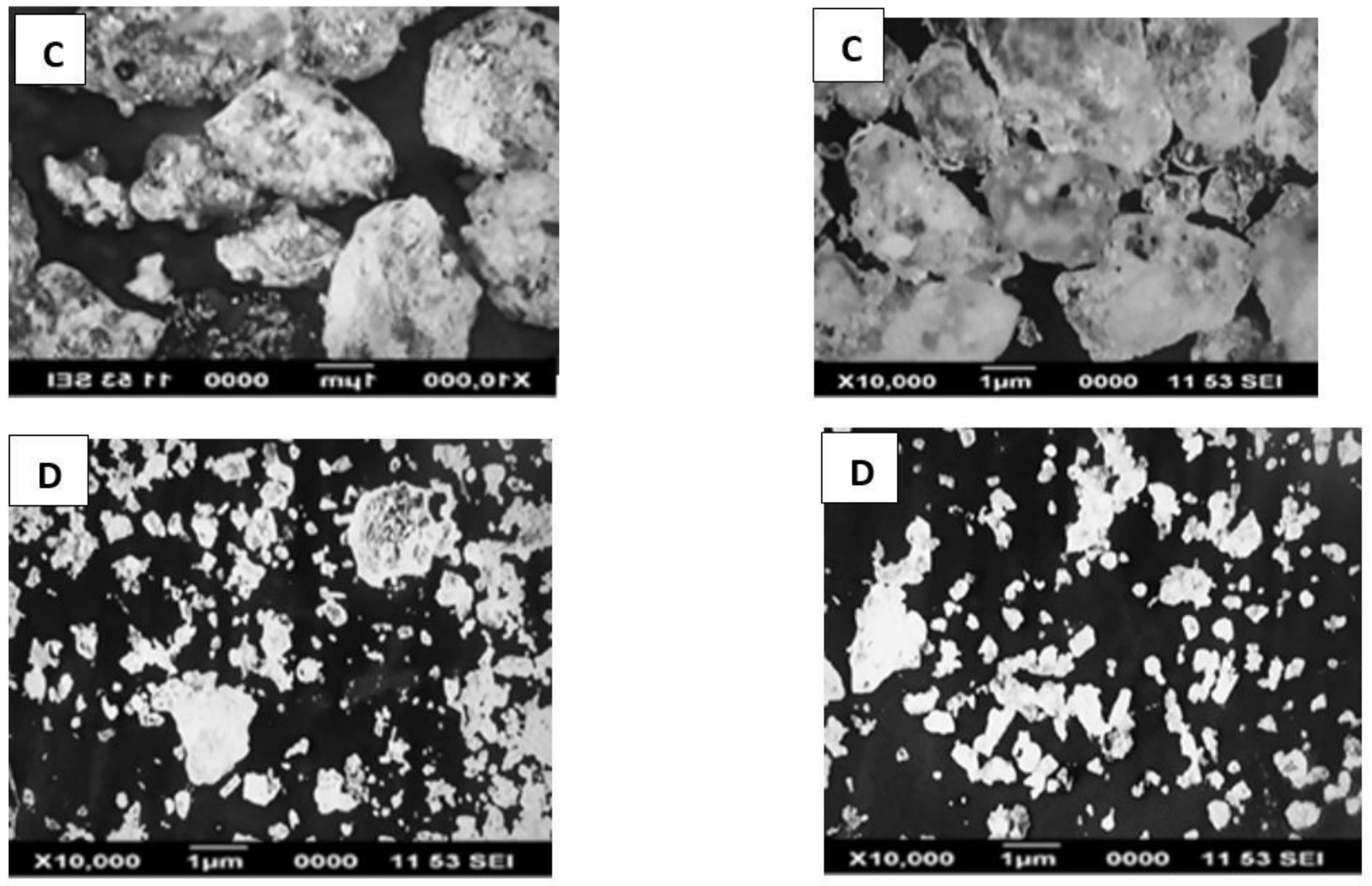

Figure 12

c. SEM Micrographs of the Polluted Soil of Angwan Kawo Before Remediation with S. acuta. d. SEM Morphological Appearance of the Remediated Soil of Angwan Kawo with S. acuta After Remediation 

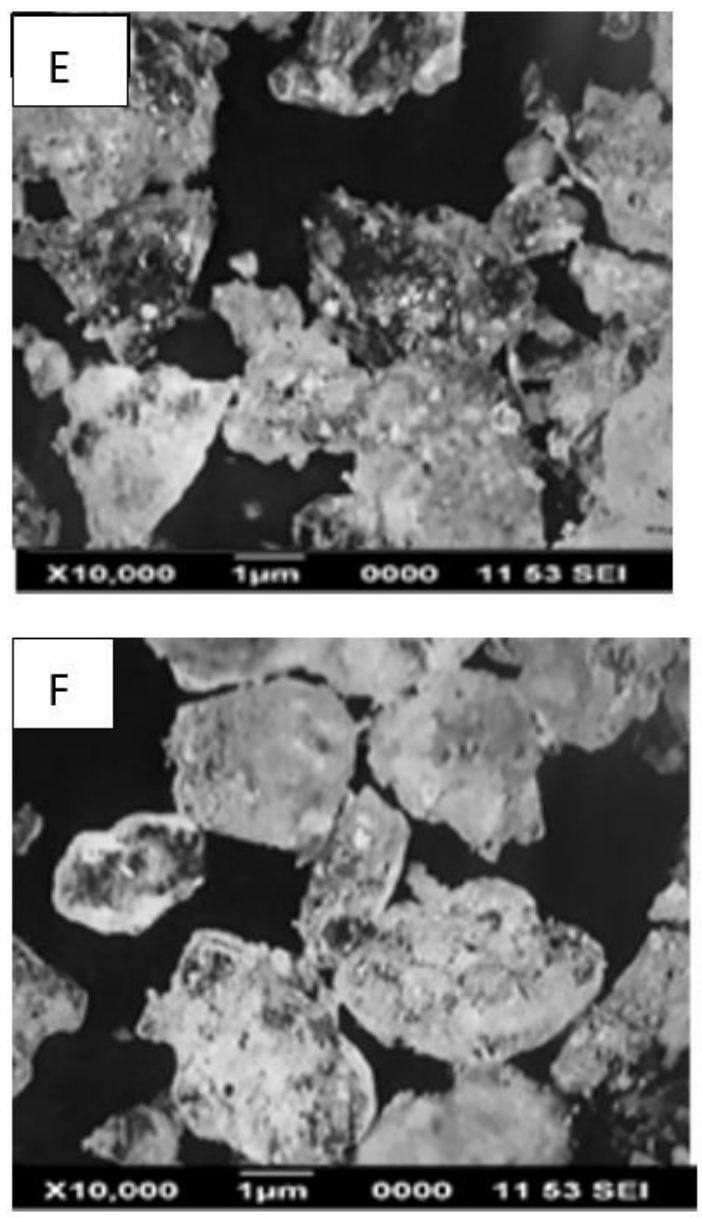
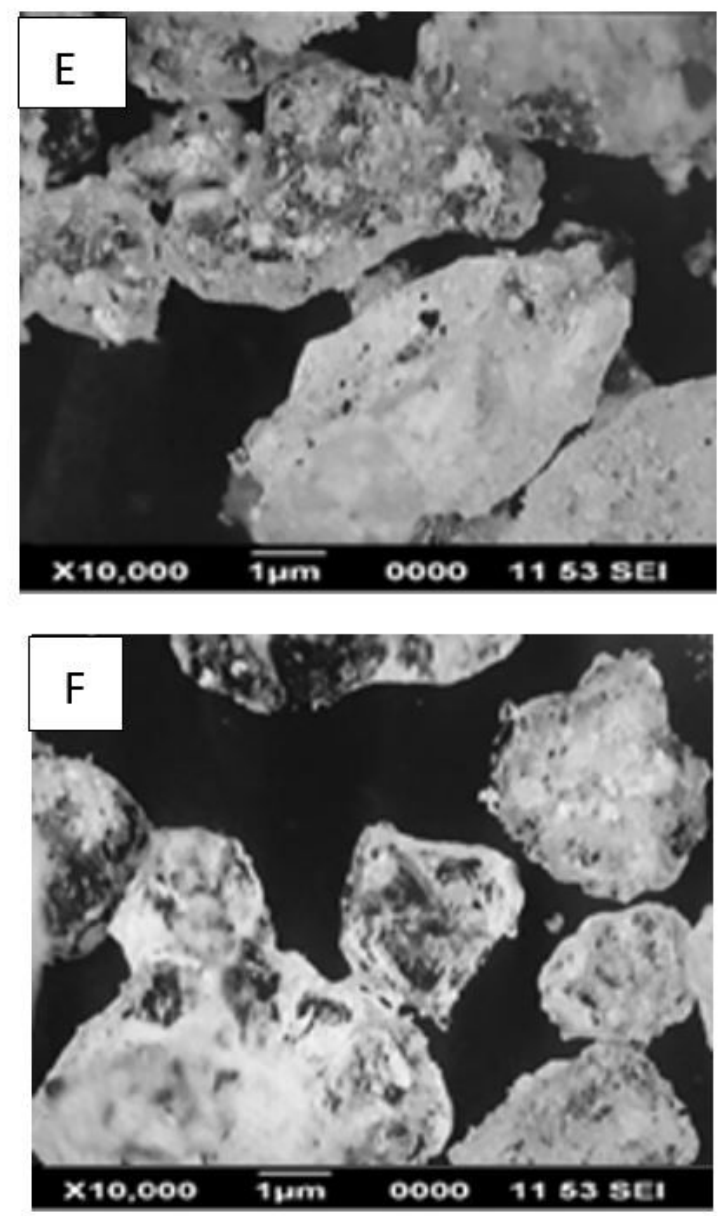

Figure 13

e. SEM Micrographs of the Polluted Soil of Angwan Magiro Before Remediation with M. officinalis L. f. SEM Morphological Appearance of the Remediated Soil of Angwan Magiro with M. officinalis $L$ After Remediation 

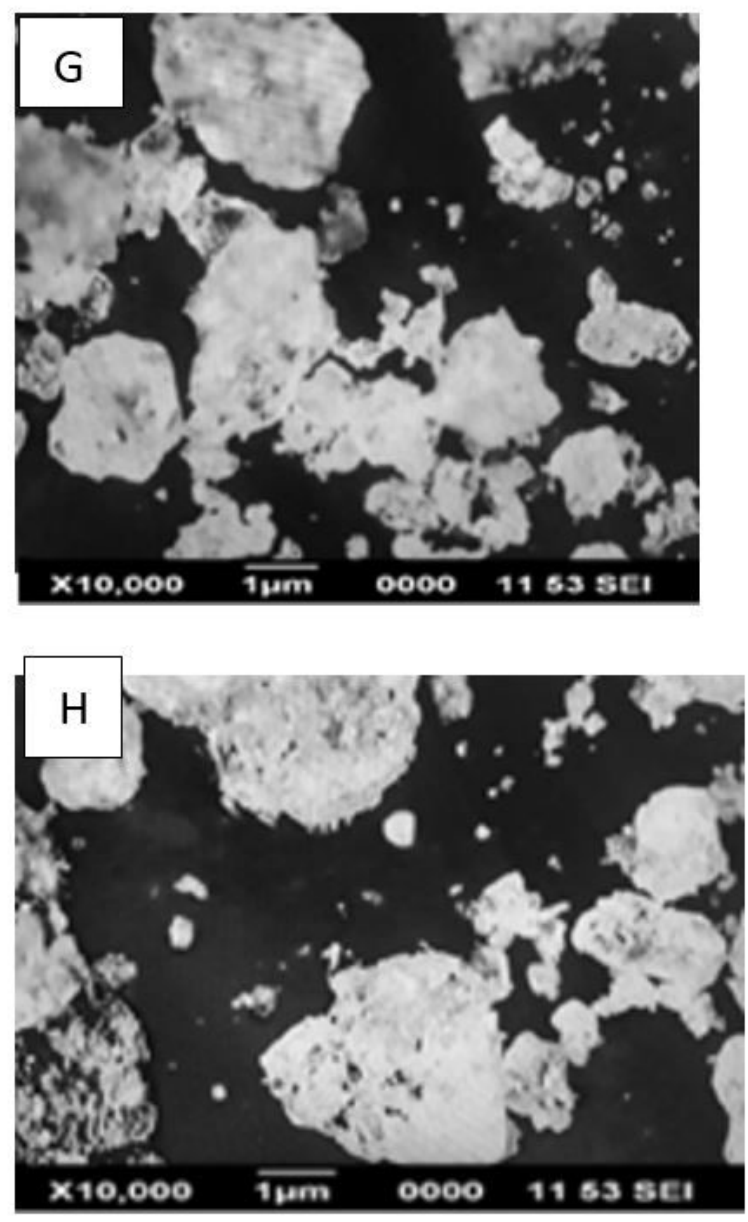
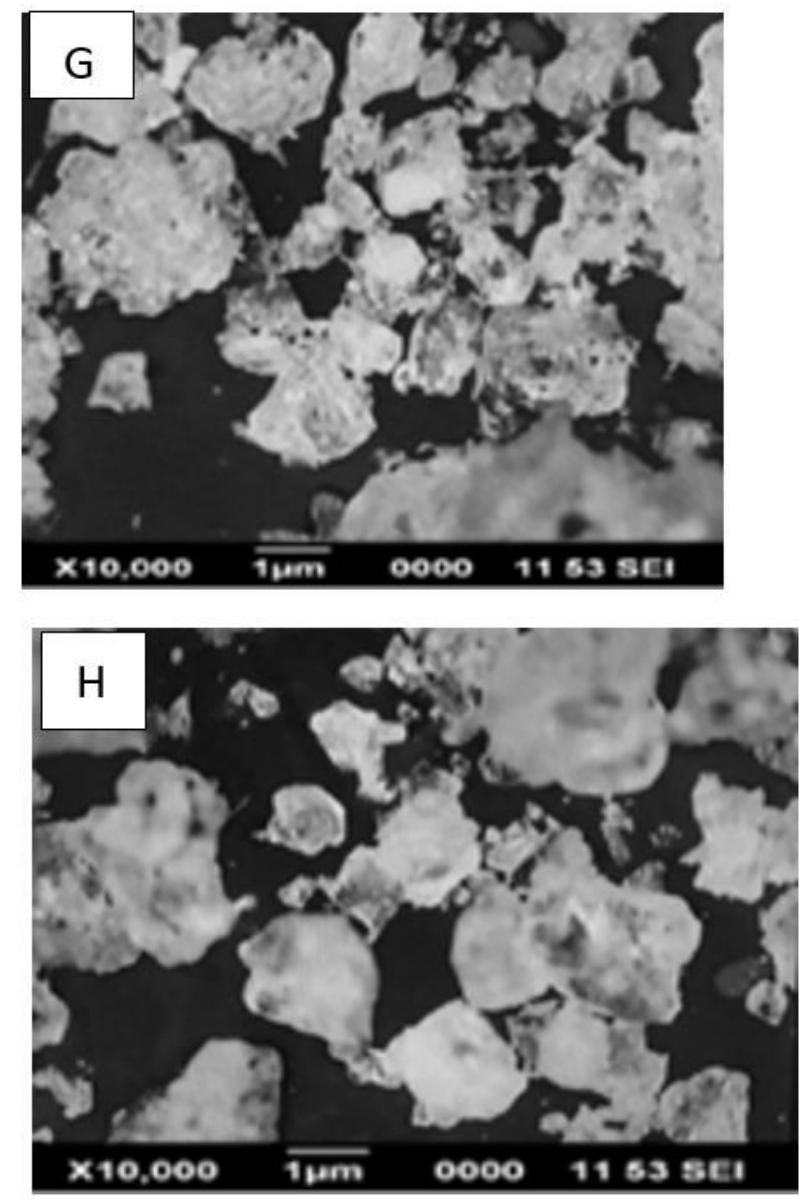

\section{Figure 14}

g. SEM Micrographs of the Polluted Soil of Angwan Magiro Before Remediation with S. acuta. h. SEM Morphological Appearance of the remediated Soil of Angwan Magiro with S. acuta 